\title{
Isomonodromic tau-functions from Liouville conformal blocks
}

\author{
N. Iorgov ${ }^{a 1}$, O. Lisovyy ${ }^{b 2}$, J. Teschner $^{c 3}$ \\ ${ }^{a}$ Bogolyubov Institute for Theoretical Physics, 03680, Kyiv, Ukraine \\ ${ }^{b}$ Laboratoire de Mathématiques et Physique Théorique, Université de Tours, 37200 Tours, France \\ ${ }^{c}$ DESY Theory, Notkestr. 85, 22603 Hamburg, Germany
}

\begin{abstract}
The goal of this note is to show that the Riemann-Hilbert problem to find multivalued analytic functions with $\mathrm{SL}(2, \mathbb{C})$-valued monodromy on Riemann surfaces of genus zero with $n$ punctures can be solved by taking suitable linear combinations of the conformal blocks of Liouville theory at $c=1$. This implies a similar representation for the isomonodromic tau-function. In the case $n=4$ we thereby get a proof of the relation between tau-functions and conformal blocks discovered in [GIL]. We briefly discuss a possible application of our results to the study of relations between certain $\mathcal{N}=2$ supersymmetric gauge theories and conformal field theory.
\end{abstract}

\section{Introduction}

The problem to describe isomonodromic deformations of ordinary differential equations has attracted a lot of attention in the past. This is due to the existence of a large number of applications in various areas of mathematics and theoretical physics, as well as the mathematical beauty and depth of the problem itself.

A first striking relation with quantum field theory was exhibited in a series of papers of Sato, Miwa and Jimbo which appeared at the end of the 1970's, see in particular [SMJ79], and [SMJ80] for a review. The results include the identification of the isomonodromic tau-functions, the generating functions for the Hamiltonians of the isomonodromic flows, with certain correlation functions in a quantum field theory of chiral free fermions.

The main result of this paper is another relation between conformal field theory and the

\footnotetext{
${ }^{1}$ iorgov@bitp.kiev.ua

${ }^{2}$ lisovyi@1mpt.univ-tours.fr

${ }^{3}$ teschner@mail.desy.de
} 
isomonodromic deformation problem: The tau-functions for isomonodromic deformations of flat SL(2)-connections on $n$-punctured spheres coincide with certain linear combinations of the Liouville conformal blocks at $c=1$. This result leads in particular to a proof of the relation between Liouville conformal blocks and the tau-function of Painlevé VI that was discovered in [GIL].

We are going to show that our result can be understood as a sort of bosonization of the fermionic representations of tau-functions. To this aim we are going to show that our construction is essentially equivalent to a bosonic construction of the so-called twist fields whose insertion generates a singularity for the fermion field with specified monodromy. In our approach the twist fields are constructed from the chiral vertex operators of the Virasoro algebra.

Expressing the isomonodromic tau-functions in terms of Liouville conformal blocks appears to have certain advantages compared to the previously known representations. The famous formula for the asymptotics of Painlevé VI found by Jimbo [Ji], for example, is an easy consequence. More generally, one may take advantage of the various results known about the Liouville conformal blocks in order to get detailed information on the isomonodromic taufunctions. Conversely, one may use this connection to find highly non-trivial new results about the Liouville conformal blocks at $c=1$ [ILT].

As an interesting application we are going to show how the known algebro-geometric solutions of the Schlesinger system on $C_{0, n}[\overline{\mathrm{KK}}]$ arise from conformal blocks of the Ashkin-Teller critical model [Za, ZZ].

In the conclusions we'll discuss a possible application of our results to the study of $\mathcal{N}=2$ supersymmetric gauge theories: They can be used to connect two recently discovered relations between certain classes of $\mathcal{N}=2, d=4$ supersymmetric gauge theories on the one hand, and two-dimensional conformal field theories on the other hand.

The paper is organised as follows. In Section 2 we review the basic formulation of the RiemannHilbert problem together with some basic material on the parameterization of monodromy groups. The following Section 3 collects the necessary background on Liouville conformal blocks. Our main result is described in Section 4, We define infinite linear combinations of the Virasoro conformal blocks, and show that the result solves the Riemann-Hilbert problem. Section 5 describes how to reformulate our results to get a bosonic construction of twist fields creating singularities for fermion fields with specified monodromy. The following Section 6 describes two applications: We first rederive Jimbo's formula for the asymptotics of Painlevé VI from our results, and show that specializing our construction to Ashkin-Teller conformal blocks reproduces the algebro-geometric solutions found in $[\mathrm{KK}]$. In the conclusions we indicate interesting directions for future research including the application to supersymmetric gauge theories mentioned above. 


\section{The Riemann-Hilbert problem}

\subsection{Formulation of the Riemann Hilbert problem}

The fundamental group $\pi_{1}$ of $C_{0, n}:=\mathbb{P}^{1} \backslash\left\{z_{1}, \ldots, z_{n}\right\}$ has $n$ generators $\chi_{1}, \ldots, \chi_{n}$ subject to one relation $\chi_{1} \circ \chi_{2} \circ \cdots \circ \chi_{n}=1$. Representations $\rho$ of $\pi_{1}\left(C_{0, n}\right)$ in $\operatorname{SL}(2, \mathbb{C})$ are specified by collections of matrices $M_{k}:=\rho\left(\chi_{k}\right) \in \mathrm{SL}(2, \mathbb{C}), k=1, \ldots, n$ satisfying $M_{n} \cdot M_{n-1} \ldots$. $M_{1}=1$ up to overall conjugation with elements of $\operatorname{SL}(2, \mathbb{C})$. We will be interested in the cases where the matrices $M_{k}$ are diagonalizable with fixed eigenvalues $e^{ \pm 2 \pi i m_{k}}$. The space of all such representations of $\pi_{1}\left(C_{0, n}\right)$ is then $2(n-3)$-dimensional.

It will be convenient to choose a base-point $y_{0}$ on $C_{0, n}$. The dependence on the choice of $y_{0}$ will turn out to be inessential. We may then represent the generators $\chi_{k}$ by closed paths starting and ending at $y_{0}$. The Riemann-Hilbert problem is to find a multivalued analytic matrix function $Y(y)$ on $C_{0, n}$ such that the monodromy along $\chi_{k}$ is represented as

$$
Y\left(\chi_{k} \cdot y\right)=Y(y) M_{k},
$$

where $Y\left(\chi_{k} . y\right)$ denotes the analytic continuation of $Y(y)$ along $\chi_{k}$.

The solution to this problem is unique up to left multiplication with single valued matrix functions. In order to fix this ambiguity we need to specify the singular behavior of $Y(y)$, leading to the following refined version of the Riemann-Hilbert problem: Find a matrix function $Y(y)$ such that the following conditions are satisfied.

i) $Y\left(y_{0}\right)=1$,

ii) $Y(y)$ is a multivalued, analytic and invertible on $C_{0, n}$,

iii) There exist neighborhoods of $z_{k}, k=1, \ldots, n$ where $Y(y)$ can be represented as

$$
Y(y)=\hat{Y}^{(k)}(y)\left(y-z_{k}\right)^{\mu_{k}}, \quad M_{k}=e^{2 \pi \mathrm{i} \mu_{k}},
$$

with $\hat{Y}^{(k)}(y)$ being holomorphic and invertible at $y=z_{k}$ and $\mu_{1}, \ldots, \mu_{n} \in \mathfrak{s l}(2, \mathbb{C})$.

If such function $Y(y)$ exists, it is uniquely determined by the monodromy data $\mu=$ $\left(\mu_{1}, \ldots, \mu_{n}\right)$.

The refined Riemann-Hilbert problem naturally arises in the study of rank 2 flat connections on $C_{0, n}$. Any flat connection on $C_{0, n}$ is gauge equivalent to a holomorphic connection of the form $\partial_{y}-A(y)$, with $A(y)$ of the form

$$
A(y)=\sum_{k=1}^{n} \frac{A_{k}}{y-z_{k}}
$$

\footnotetext{
${ }^{4}$ Here understood as anti-homomorphisms $\rho: \pi_{1}\left(C_{0, n}\right) \rightarrow \mathrm{SL}(2, \mathbb{C})$
} 
where $A_{1}, \ldots A_{n} \in \mathfrak{s l}(2, \mathbb{C}), \sum_{k=1}^{n} A_{k}=0$. One may then consider the fundamental matrix solution $Y(y)$ of the differential equation

$$
\frac{\partial}{\partial y} Y(y)=A(y) Y(y)
$$

normalized by $Y\left(y_{0}\right)=1$. It will automatically satisfy ii) and iii) for certain $\mu_{1}, \ldots, \mu_{n}$, provided that the eigenvalues $\pm m_{k}$ of $A_{k}$ satisfy the condition $2 m_{k} \notin \mathbb{Z}$. Any representation $\rho$ : $\pi_{1}\left(C_{0, n}\right) \rightarrow \mathrm{SL}(2, \mathbb{C})$ can be realized as monodromy representation of such a Fuchsian system, which means that a solution to the Riemann-Hilbert problem formulated will generically exist. The Riemann-Hilbert correspondence between flat connections $\partial_{y}-A(y)$ and representations $\rho: \pi_{1}\left(C_{0, n}\right) \rightarrow \operatorname{SL}(2, \mathbb{C})$ allows us to identify the moduli space $\mathcal{M}_{\text {flat }}\left(C_{0, n}\right)$ of flat $\mathfrak{s l}(2, \mathbb{C})$ connections on $C_{0, n}$ with the so-called character variety $\operatorname{Hom}\left(\pi_{1}\left(C_{0, n}\right), \operatorname{SL}(2, \mathbb{C})\right) / \mathrm{SL}(2, \mathbb{C})$.

\subsection{Trace coordinates}

Useful sets of coordinates for $\mathcal{M}_{\text {flat }}\left(C_{0, n}\right)$ are given by the trace functions $L_{\gamma}:=\operatorname{tr} \rho(\gamma)$ associated to any simple closed curve $\gamma$ on $C_{0, n}$. Minimal sets of trace functions that can be used to parameterize $\mathcal{M}_{\text {flat }}\left(C_{0, n}\right)$ can be identified using pants decompositions. In order to have uniform notations, let us replace the punctures $z_{1}, \ldots, z_{n}$ by little holes obtained by cutting along non-intersecting simple closed curves $\delta_{k}$ surrounding the punctures $z_{k}, k=1, \ldots, n$, respectively. A pants decomposition is defined by cutting $C_{0, n}$ along $n-3$ simple closed curves $\gamma_{r}$, $r=1, \ldots, n-3$ on $C_{0, n}$. This will decompose $C_{0, n}$ into a disjoint union of $n-2$ three-holed spheres $C_{0,3}^{t}, t=1, \ldots, n-2$. The collection $\mathcal{C}=\left\{\gamma_{1}, \ldots, \gamma_{n-3}\right\}$ of curves will be called the cut system.

To each curve $\gamma_{r} \in \mathcal{C}$ let us associate the union of the two three-holed spheres that have $\gamma_{r}$ in its boundary, a four-holed sphere $C_{0,4}^{r}$. It will be assumed that the curves $\gamma_{r}, r=1, \ldots, n-3$ are oriented. The orientation of $\gamma_{r}$ allows us to introduce a natural numbering of the boundaries of $C_{0,4}^{r}$. We may then consider the curves $\gamma_{s}^{r}$ and $\gamma_{t}^{r}$ which encircle the pairs of boundary components of $C_{0,4}^{r}$ with numbers $(1,2)$ and $(2,3)$, respectively. The corresponding trace functions will be denoted as $L_{s}^{r}$ and $L_{t}^{r}$. The collection of pairs of trace functions $\left(L_{s}^{r}, L_{t}^{r}\right), r=1, \ldots, n-3$ can be used to parameterize $\mathcal{M}_{\text {flat }}\left(C_{0, n}\right)$.

A closely related set of coordinates for $\mathcal{M}_{\text {flat }}\left(C_{0, n}\right)$ is obtained by parameterizing $L_{s}^{r}$ and $L_{t}^{r}$ in terms of complex numbers $\left(\sigma_{r}, \tau_{r}\right)$ as

$$
\begin{aligned}
L_{s}^{r} & =2 \cos 2 \pi \sigma_{r} \\
\left(\sin \left(2 \pi \sigma_{r}\right)\right)^{2} L_{t}^{r} & =C_{+}\left(\sigma_{r}\right) e^{i \tau_{r}}+C_{0}\left(\sigma_{r}\right)+C_{-}\left(\sigma_{r}\right) e^{-i \tau_{r}},
\end{aligned}
$$


where

$$
\begin{aligned}
C_{+}\left(\sigma_{r}\right)= & -4 \prod_{s= \pm 1} \sin \pi\left(\sigma_{r}+s\left(\sigma_{1}^{r}-\sigma_{2}^{r}\right)\right) \sin \pi\left(\sigma_{r}+s\left(\sigma_{3}^{r}-\sigma_{4}^{r}\right)\right), \\
C_{0}\left(\sigma_{r}\right)= & 2\left[\cos 2 \pi \sigma_{2}^{r} \cos 2 \pi \sigma_{3}^{r}+\cos 2 \pi \sigma_{1}^{r} \cos 2 \pi \sigma_{4}^{r}\right] \\
& -2 \cos 2 \pi \sigma_{r}\left[\cos 2 \pi \sigma_{1}^{r} \cos 2 \pi \sigma_{3}^{r}+\cos 2 \pi \sigma_{2}^{r} \cos 2 \pi \sigma_{4}^{r}\right], \\
C_{-}\left(\sigma_{r}\right)= & -4 \prod_{s= \pm 1} \sin \pi\left(\sigma_{r}+s\left(\sigma_{1}^{r}+\sigma_{2}^{r}\right)\right) \sin \pi\left(\sigma_{r}+s\left(\sigma_{3}^{r}+\sigma_{4}^{r}\right)\right) .
\end{aligned}
$$

In order to define $\sigma_{i}^{r}, i=1, \ldots, 4$ in (2.6) let us note that the boundary of $C_{0,4}^{r}$ with label $i$ may either be a curve $\gamma_{r^{\prime}} \in \mathcal{C}$, or it must coincide with a curve $\delta_{k}$ surrounding puncture $z_{k}$. We will identify $\sigma_{i}^{r} \equiv \sigma_{r^{\prime}}$ in the first case, while $\sigma_{i}^{r}$ will be identified with an eigenvalue of $\mu_{k}$ otherwise. The collection of data $\left(\sigma_{r}, \tau_{r}\right), r=1, \ldots, n-3$ will be denoted as $(\sigma, \tau)$. We observe that the coordinates $(\sigma, \tau)$ are for $n=4$ close relatives of the parameters used in [Ji]]. They are also closely related to the coordinates used in [NRS].

\subsection{Isomonodromic deformations and tau-function}

Let us briefly recall the well-known relations to the isomonodromic deformation problem. Given a solution $Y(y)$ to the Riemann-Hilbert problem we may define an associated connection $A(y)$ as

$$
A(y) \equiv A(y \mid z):=\left(\partial_{y} Y(y)\right) \cdot(Y(y))^{-1},
$$

It follows from (2.2) that

$$
A(y \mid z)=\sum_{k=1}^{n} \frac{A_{k}(z)}{y-z_{k}} .
$$

It is well-known that variations of the positions $z_{r}$ will not change the monodromies of the connection $A(y)$ provided that the matrix residues $A_{k}=A_{k}(z)$ satisfy the following equations,

$$
\begin{aligned}
\partial_{z_{k}} A_{k} & =-\sum_{l \neq k} \frac{\left[A_{k}, A_{l}\right]}{z_{k}-z_{l}}, \\
\partial_{z_{l}} A_{k} & =\frac{y_{0}-z_{k}}{y_{0}-z_{l}} \frac{\left[A_{k}, A_{l}\right]}{z_{k}-z_{l}}, \quad k \neq l,
\end{aligned}
$$

In the limit $y_{0} \rightarrow \infty$ one finds the Schlesinger equations

$$
\begin{aligned}
\partial_{z_{k}} A_{k} & =-\sum_{l \neq k} \frac{\left[A_{k}, A_{l}\right]}{z_{k}-z_{l}}, \\
\partial_{z_{l}} A_{k} & =\frac{\left[A_{k}, A_{l}\right]}{z_{k}-z_{l}}, \quad k \neq l .
\end{aligned}
$$


The Schlesinger equations define Hamiltonian flows, generated by the Hamiltonians

$$
H_{k}:=\frac{1}{2} \operatorname{res}_{y=z_{k}} \operatorname{tr} A^{2}(y)=\sum_{l \neq k} \frac{\operatorname{tr}\left(A_{k} A_{l}\right)}{z_{l}-z_{k}},
$$

using the Poisson structure

$$
\left\{A(y) \stackrel{\otimes}{,} A\left(y^{\prime}\right)\right\}=\left[\frac{\mathcal{P}}{y-y^{\prime}}, A(y) \otimes 1+1 \otimes A\left(y^{\prime}\right)\right],
$$

where $\mathcal{P}$ denotes the permutation matrix. The tau-function $\tau(z)$ is defined as the generating function for the Hamiltonians $H_{k}$,

$$
H_{k}=\partial_{z_{k}} \log \tau(z) .
$$

Integrability of (2.13) is ensured by the Schlesinger equations (2.10).

\section{Chiral vertex operators and conformal blocks}

Let us introduce the necessary definitions and results on the representation theory of the Virasoro algebra which has generators $L_{n}, n \in \mathbb{Z}$ and relations

$$
\left[L_{n}, L_{m}\right]=(n-m) L_{n+m}+\frac{c}{12} n\left(n^{2}-1\right) \delta_{n+m, 0} .
$$

Although we will ultimately be interested in the case $c=1$, it will be useful to consider more general values of $c$ in some of our arguments. Highest weight representations $\mathcal{V}_{\alpha}$ are generated from vectors $|\alpha\rangle$ which satisfy

$$
L_{n}|\alpha\rangle=0, \quad n>0, \quad L_{0}|\alpha\rangle=\Delta_{\alpha}|\alpha\rangle,
$$

where $\Delta_{\alpha}=\alpha(Q-\alpha)$ if $c$ is parameterized as $c=1+6 Q^{2}$. The representations $\mathcal{V}_{\alpha}$ can be decomposed into the so-called energy-eigenspaces

$$
\mathcal{V}_{\alpha} \simeq \bigoplus_{n \in \mathbb{Z}_{\geq 0}} \mathcal{V}_{\alpha}^{(n)}
$$

defined by the condition $L_{0} v=\left(\Delta_{\alpha}+n\right) v$ for all $v \in \mathcal{V}_{\alpha}^{(n)}$.

\subsection{Chiral vertex operators}

Chiral vertex operators $V_{\beta_{2} \beta_{1}}^{\alpha}(z)$ can be defined as operators that map $\mathcal{V}_{\beta_{1}} \rightarrow \mathcal{V}_{\beta_{2}}$ such that

$$
L_{n} V_{\beta_{2} \beta_{1}}^{\alpha}(z)-V_{\beta_{2} \beta_{1}}^{\alpha}(z) L_{n}=z^{n}\left(z \partial_{z}+\Delta_{\alpha}(n+1)\right) V_{\beta_{2} \beta_{1}}^{\alpha}(z) .
$$


We have in particular

$$
V_{\beta_{2} \beta_{1}}^{\alpha}(z)\left|\beta_{1}\right\rangle=N\left(\beta_{2}, \alpha, \beta_{1}\right) z^{\Delta_{\beta_{2}}-\Delta_{\beta_{1}}-\Delta_{\alpha}}\left[\left|\beta_{2}\right\rangle+\mathcal{O}(z)\right]
$$

with a normalization factor $N\left(\beta_{2}, \alpha, \beta_{1}\right)$ that will be specified later. It is well-known that the conditions (3.17) define $z^{\Delta_{\beta_{1}}+\Delta_{\alpha}-\Delta_{\beta_{2}}} V_{\beta_{2} \beta_{1}}^{\alpha}(z)$ uniquely in the sense of formal power series in $z$,

$$
V_{\beta_{2} \beta_{1}}^{\alpha}(z)=z^{\Delta_{\beta_{2}}-\Delta_{\beta_{1}}-\Delta_{\alpha}} \sum_{n=-\infty}^{\infty} z^{n} W_{\beta_{2} \beta_{1}}^{\alpha}(n), \quad W_{\beta_{2} \beta_{1}}^{\alpha}(n): \mathcal{V}_{\beta_{1}}^{(k)} \rightarrow \mathcal{V}_{\beta_{2}}^{(k+n)}
$$

It has furthermore been argued in [T03] that the composition $V_{\beta_{3} \beta_{2}}^{\alpha_{2}}(z) V_{\beta_{2} \beta_{1}}^{\alpha_{1}}(w)$ of such vertex operators exists for $|w / z|<1$, and that matrix elements such as

$$
\left\langle\alpha_{n}\left|V_{\alpha_{n} \beta_{n-3}}^{\alpha_{n-1}}\left(z_{n-1}\right) V_{\beta_{n-3} \beta_{n-4}}^{\alpha_{n-2}}\left(z_{n-2}\right) \cdots V_{\beta_{1} \alpha_{1}}^{\alpha_{2}}\left(z_{2}\right)\right| \alpha_{1}\right\rangle
$$

are represented by absolutely convergent power series in $z_{k} / z_{k+1}, k=2, \ldots, n-2$.

From each chiral vertex operator $V_{\beta_{2} \beta_{1}}^{\alpha}(z)$ one may generate a family of vertex operators called descendants of $V_{\beta_{2} \beta_{1}}^{\alpha}(z)$. The descendants of $V_{\beta_{2} \beta_{1}}^{\alpha}(z)$ are labelled by the vectors in $\mathcal{V}_{\alpha}$, and the descendant corresponding to $v \in \mathcal{V}_{\alpha}$ will be denoted as $V_{\beta_{2} \beta_{1}}^{\alpha}[v](z)$. The descendants may be defined by means of the recursion relations

$$
\begin{aligned}
& V_{\beta_{2} \beta_{1}}^{\alpha}[|\alpha\rangle](z) \equiv V_{\beta_{2} \beta_{1}}^{\alpha}(z) \\
& V_{\beta_{2} \beta_{1}}^{\alpha}\left[L_{-1} v\right](z) \equiv \partial_{z} V_{\beta_{2} \beta_{1}}^{\alpha}[v](z) \\
& V_{\beta_{2} \beta_{1}}^{\alpha}\left[L_{-2} v\right](z) \equiv: T(z) V_{\beta_{2} \beta_{1}}^{\alpha}[v](z):
\end{aligned}
$$

where the following notation has been used in (3.21c):

$$
: T(z) V_{\beta_{2} \beta_{1}}^{\alpha}[v](z): \equiv \sum_{k<-1} z^{-k-2} L_{k} V_{\beta_{2} \beta_{1}}^{\alpha}[v](z)+\sum_{k \geq-1} z^{-k-2} V_{\beta_{2} \beta_{1}}^{\alpha}[v](z) L_{k} .
$$

The recursion relations (3.21) suffice to define $V_{\beta_{2} \beta_{1}}^{\alpha}\left[L_{-n} v\right](z)$ for all $n>0$ thanks to the Virasoro algebra (3.14).

Using the descendants one may define a trilinear form $\mathcal{C}_{0,3}: \mathcal{V}_{\alpha_{3}} \otimes \mathcal{V}_{\alpha_{2}} \otimes \mathcal{V}_{\alpha_{1}} \rightarrow \mathbb{C}$ as

$$
\mathcal{C}_{0,3}\left(v_{3} \otimes v_{2} \otimes v_{1}\right):=\left\langle v_{3}\left|V_{\alpha_{3} \alpha_{1}}^{\alpha_{2}}\left[v_{2}\right](z)\right| v_{1}\right\rangle
$$

This trilinear form can be identified with the conformal block associated to the three-punctured sphere $C_{0,3}$.

The definition of descendants allows us to introduce another way to compose chiral vertex operators. We may e.g. consider

$$
V_{\beta_{2} \beta_{1}}^{\beta_{3}}\left[V_{\beta_{3} \alpha_{1}}^{\alpha_{2}}\left[v_{2}\right](w-z) v_{1}\right](z)
$$


which is defined a priori as a formal power series in $w-z$. Quadrilinear forms such as

$$
\mathcal{C}_{0,4}\left(v_{4} \otimes \ldots \otimes v_{1}\right):=\left\langle v_{4}\left|V_{\alpha_{4} \alpha_{1}}^{\beta}\left[V_{\beta \alpha_{2}}^{\alpha_{3}}\left[v_{3}\right](w-z) v_{2}\right](z)\right| v_{1}\right\rangle,
$$

will define absolutely convergent series in $w-z$ for all $v_{4}, \ldots, v_{1}$ of finite energy. The quadrilinear forms $\mathcal{C}_{0,4}\left(v_{4} \otimes \ldots \otimes v_{1}\right)$ can be identified with conformal blocks associated to the fourpunctured sphere $C_{0,4}$.

By using the two types of composition of chiral vertex operators introduced above one may construct conformal blocks associated to arbitrary pants decompositions of $n$-punctured spheres.

\subsection{Degenerate fields}

Of particular importance for us will be the special case where $\alpha=-b / 2$, assuming that $Q$ is represented as $Q=b+b^{-1}$. If furthermore $\beta_{2}$ and $\beta_{1}$ are related as $\beta_{2}=\beta_{1} \mp b / 2$, the vertex operators $\psi_{s}(y) \equiv \psi_{\beta_{1}, s}(y):=V_{\beta_{1}-s b / 2, \beta_{1}}^{-b / 2}(y), s= \pm 1$, are well-known to satisfy a differential equation of the form

$$
\partial_{y}^{2} \psi_{\beta_{1}, s}(y)+b^{2}: T(y) \psi_{\beta_{1}, s}(y):=0,
$$

with normal ordering defined in (3.22). The chiral vertex operators $\psi_{\beta_{1}, s}(y)$ are called degenerate fields. It follows from (3.26) that matrix elements such as

$$
\begin{aligned}
& \mathcal{F}\left(\alpha ; \beta|z| y_{0} \mid y\right):=\left\langle\alpha_{n}\left|\psi_{s^{\prime}}\left(y_{0}\right) \psi_{s}(y)\right| \Theta\right\rangle, \\
& |\Theta\rangle:=V_{\alpha_{n}+\left(s+s^{\prime}\right) \frac{b}{2}, \beta_{n-3}}^{\alpha_{n-1}}\left(z_{n-1}\right) V_{\beta_{n-3} \beta_{n-4}}^{\alpha_{n-2}}\left(z_{n-2}\right) \cdots V_{\beta_{1} \alpha_{1}}^{\alpha_{2}}\left(z_{2}\right) V_{\alpha_{1}, 0}^{\alpha_{1}}\left(z_{1}\right)|0\rangle,
\end{aligned}
$$

will satisfy the partial differential equation $\mathcal{D}_{\mathrm{BPZ}} \mathcal{F}=0$, with

$$
\mathcal{D}_{\mathrm{BPZ}}:=\frac{1}{b^{2}} \frac{\partial^{2}}{\partial y^{2}}+\frac{\Delta_{-\frac{b}{2}}}{\left(y-y_{0}\right)^{2}}+\frac{1}{y-y_{0}} \frac{\partial}{\partial y_{0}}+\sum_{k=1}^{n-1}\left(\frac{\Delta_{\alpha_{k}}}{\left(y-z_{k}\right)^{2}}+\frac{1}{y-z_{k}} \frac{\partial}{\partial z_{k}}\right),
$$

together with a similar differential equation for $y_{0}$. Using this differential equation it may be shown that $\mathcal{F}\left(\alpha ; \beta|z| y_{0} \mid y\right)$, considered as a function of $y$, can be analytically continued to a multivalued analytic function on $C_{0, n}$.

\subsection{Braiding and fusion of degenerate fields}

The differential equations (3.26) satisfied by the degenerate fields can be used to get a precise description of the monodromies of the conformal blocks $\mathcal{F}\left(\alpha ; \beta|z| y_{0} \mid y\right)$ defined in (3.27). Let us briefly summarize the relevant results. There are three ways to compose a degenerate field with a generic chiral vertex operator,

$$
\begin{aligned}
& \text { (1) } V_{\alpha_{3}, \alpha_{1}-s \frac{b}{2}}^{\alpha_{2}}\left[v_{2}\right](z) \psi_{s}(y), \\
& \text { (3) } \psi_{-s}(y) V_{\alpha_{3}-s \frac{b}{2}, \alpha_{1}}^{\alpha_{2}}\left[v_{2}\right](z),
\end{aligned}
$$$$
\text { (2) } V_{\alpha_{3}, \alpha_{1}}^{\alpha_{2}-\frac{b}{2}}\left[\psi_{s}(y-z) v_{2}\right](z) \text {. }
$$ 


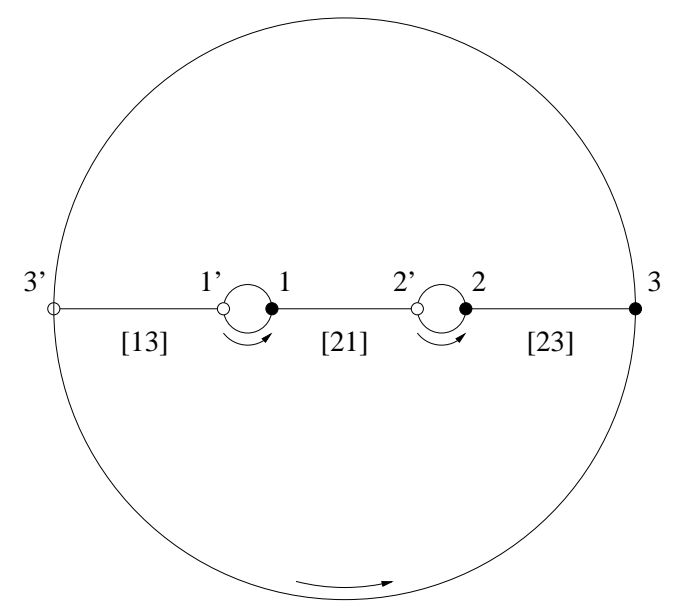

Figure 1: A sphere with three holes. The arrows indicate our orientation conventions.

The three ways (3.29) to compose these vertex operators correspond to having the degenerate field $\psi_{s}(y)$ located in the vicinity of the boundary components with labels 1, 2 and 3, respectively, referring to Figure 1 for the notations. The conformal blocks defined using the three compositions (3.29) are single valued and analytic in neighborhoods of the black dots marked in Figure 1 on the boundaries of the three holes of $C_{0,3}$, respectively. We are going to describe their analytic continuation to the universal cover of $C_{0,3}$. It will be helpful to introduce a separate notation for the vertex operator $\psi_{s}(y)$ when it is inserted at the antipodal point of the circle $|y|=$ const,

$$
\psi_{\beta, s}^{\prime}(y)=B_{s}(\beta) \psi_{\beta, s}\left(e^{-\pi i} y\right), \quad B_{s}(\alpha)=e^{\pi i\left(\Delta_{\alpha-s \frac{b}{2}}-\Delta_{\alpha}-\Delta_{-\frac{b}{2}}\right)} .
$$

The vertex operators $\psi_{\beta, s}^{\prime}(y)$ are single-valued in an open neighborhood containing segments of the negative real axis. One may naturally consider compositions (1)'-(3)' of the form (3.29), but with $\psi_{s}(y)$ replaced by $\psi_{s}^{\prime}(y)$. Regions on $C_{0,3}$ where the compositions (1)'-(3)' define singlevalued analytic conformal blocks are neighbourhoods of the small empty circles in Figure 1.

The main building block for the monodromies will be the following relations,

$$
\begin{aligned}
\psi_{-s_{1}}(y) V_{\alpha_{3}-s_{1} \frac{b}{2}, \alpha_{1}}^{\alpha_{2}}\left[v_{2}\right](z) & =\sum_{s_{2}= \pm 1} F_{s_{1}, s_{2}}^{[23]} V_{\alpha_{3}, \alpha_{1}}^{\alpha_{2}-s_{2} \frac{b}{2}}\left[\psi_{s_{2}}(y-z) v_{2}\right](z), \\
V_{\alpha_{3}, \alpha_{1}-s_{1} \frac{b}{2}}^{\alpha_{2}}\left[v_{2}\right](z) \psi_{s_{1}}(y) & =\sum_{s_{2}= \pm 1} F_{s_{1}, s_{2}}^{[21]} V_{\alpha_{3}, \alpha_{1}}^{\alpha_{2}-s_{2} \frac{b}{2}}\left[\psi_{s_{2}}^{\prime}(y-z) v_{2}\right](z), \\
\psi_{-s_{1}}^{\prime}(y) V_{\alpha_{3}-s_{1} \frac{b}{2}, \alpha_{1}}^{\alpha_{2}}\left[v_{2}\right](z) & =\sum_{s_{2}= \pm 1} F_{s_{1}, s_{2}}^{[13]} V_{\alpha_{3}, \alpha_{1}-s_{2} \frac{b}{2}}^{\alpha_{2}}\left[v_{2}\right](z) \psi_{s_{2}}^{\prime}(y) .
\end{aligned}
$$

The relevant transport matrices are given respectively as

$$
F_{s_{1}, s_{2}}^{[j i]}=\frac{\Gamma\left(1+s_{1} b\left(2 \alpha_{i}-Q\right)\right) \Gamma\left(s_{2} b\left(Q-2 \alpha_{j}\right)\right)}{\prod_{s_{3}= \pm} \Gamma\left(\frac{1}{2}+s_{1} b\left(\alpha_{i}-Q / 2\right)-s_{2} b\left(\alpha_{j}-Q / 2\right)+s_{3} b\left(\alpha_{k}-Q / 2\right)\right)},
$$

valid if the vertex operators $V_{\beta_{2}, \beta_{1}}^{\alpha}(z)$ are normalized via (3.18) with $N\left(\alpha_{3}, \alpha_{2}, \alpha_{1}\right) \equiv 1$. 
Remark 1. Comparing with the Moore-Seiberg formalism let us note that

$$
\begin{aligned}
& F_{s_{1}, s_{2}}^{[21]} \equiv F\left[\begin{array}{cc}
\alpha_{2} & -b / 2 \\
\alpha_{3} & \alpha_{1}
\end{array}\right]_{s_{1} s_{2}} \equiv F_{\alpha_{1}-s_{1} \frac{b}{2} ; \alpha_{2}-s_{2} \frac{b}{2}}\left[\begin{array}{cc}
\alpha_{2} & -b / 2 \\
\alpha_{3} & \alpha_{1}
\end{array}\right], \\
& F_{s_{1}, s_{2}}^{[23]} \equiv F\left[\begin{array}{cc}
-b / 2 & \alpha_{2} \\
\alpha_{3} & \alpha_{1}
\end{array}\right]_{s_{1} s_{2}} \equiv F_{\alpha_{3}-s_{1} \frac{b}{2} ; \alpha_{2}-s_{2} \frac{b}{2}}\left[\begin{array}{cc}
-b / 2 & \alpha_{2} \\
\alpha_{3} & \alpha_{1}
\end{array}\right], \\
& F_{s_{1}, s_{2}}^{[13]} \equiv F\left[\begin{array}{cc}
\alpha_{3} & \alpha_{2} \\
-b / 2 & \alpha_{1}
\end{array}\right]_{s_{1} s_{2}} \equiv F_{\alpha_{3}-s_{1} \frac{b}{2} ; \alpha_{1}-s_{2} \frac{b}{2}}\left[\begin{array}{cc}
\alpha_{3} & \alpha_{2} \\
-b / 2 & \alpha_{1}
\end{array}\right],
\end{aligned}
$$

The relevant fusion matrices are related to each other by the symmetries

$$
F\left[\begin{array}{cc}
\alpha_{2} & -b / 2 \\
\alpha_{3} & \alpha_{1}
\end{array}\right]_{s_{1} s_{2}}=F\left[\begin{array}{cc}
-b / 2 & \alpha_{2} \\
\alpha_{1} & \alpha_{3}
\end{array}\right]_{s_{1} s_{2}}=F\left[\begin{array}{cc}
\alpha_{3} & \alpha_{1} \\
\alpha_{2} & -b / 2
\end{array}\right]_{s_{1} s_{2}}
$$

together with

$$
F\left[\begin{array}{cc}
\alpha_{2} & -b / 2 \\
\alpha_{3} & \alpha_{1}
\end{array}\right]^{-1}=F\left[\begin{array}{cc}
\alpha_{1} & -b / 2 \\
\alpha_{3} & \alpha_{2}
\end{array}\right] .
$$

The definition of the antipodal vertex operators $\psi_{\beta, s}^{\prime}(y)$ in (3.30) is related to the elementary braid relation

$$
\left[V_{\alpha_{3}, \alpha_{1}}^{\alpha_{2}}(y) V_{\alpha_{1}, 0}^{\alpha_{1}}(z)|0\rangle\right]_{\circlearrowleft}=\Omega_{\alpha_{2}, \alpha_{1}}^{\alpha_{3}} V_{\alpha_{3}, \alpha_{2}}^{\alpha_{1}}(z) V_{\alpha_{2}, 0}^{\alpha_{2}}(y)|0\rangle,
$$

with left hand side defined by means of analytic continuation making $y$ encircle $z$ in the anticlockwise sense. It is easy to see that the "half-monodromy" used in (3.30) is related to the composition of analytic continuation (3.36) with a suitable translation. It follows that the braiding phase factor $B_{s}(\alpha)$ is related to the factors $\Omega_{\alpha_{2}, \alpha_{1}}^{\alpha_{3}}$ in (3.36) as $B_{s}(\alpha)=\Omega_{-b / 2, \alpha}^{\alpha-s b / 2}$.

In the normalisation where $N\left(\alpha_{3}, \alpha_{2}, \alpha_{1}\right) \equiv 1$ one may observe that the conformal blocks and the fusion matrices $F^{[j i]}$ are perfectly analytic with respect to the central charge $c$. We may in particular take the limit $c \rightarrow 1$ without encountering any problem. This is not the case for the kernel of the integral transformation relating conformal blocks associated to different pants decompositions.

\subsection{Monodromy action on spaces of conformal blocks}

Using these ingredients it is straightforward to show that the analytic continuation of the matrix elements $\mathcal{F}\left(\alpha ; \beta|z| y_{0} \mid y\right)$ along the closed paths $\chi_{k}$ can be expressed as a linear combination of the matrix elements $\mathcal{F}\left(\alpha ; \beta^{\prime}|z| y_{0} \mid y\right)$ having parameters $\beta_{r}^{\prime}$ that differ from $\beta_{r}$ by integer multiples of the parameter $b$. In order to have a convenient notation let us define the shift operators $\mathrm{V}_{\beta_{r}}^{\frac{1}{2}}$ which acts on functions to the left as

$$
\mathcal{F}\left(\alpha ; \beta|z| y_{0} \mid y\right) \cdot \bigvee_{\beta_{r}}^{\frac{1}{2}}=\mathcal{F}\left(\alpha ; \beta-\frac{b}{2} e_{r}|z| y_{0} \mid y\right),
$$

where $e_{r}$ is the vector in $\mathbb{C}^{n-3}$ with components $\delta_{r s}$. 


\subsubsection{Geometrical set-up}

It will be useful for us to refine the pants decompositions as follows. On each curve $\gamma$ in the extended cut system $\hat{\mathcal{C}}:=\left\{\gamma_{1}, \ldots, \gamma_{2 n-3}\right\}$, where $\gamma_{n-3+k}:=\delta_{k}$ for $k=1, \ldots, n$ let us mark two points, a black one and a white one. On each pair of pants with label $t$ let us introduce a collection of two non-intersecting arcs $[23]_{t}$, and $[13]_{t}$ that connect marked points on the boundary components labelled by 1,2 and 3, respectively. These contours are depicted in Figure 1

Let us next note that any generator $\chi_{k}$ of $\pi_{1}\left(C_{0, n}\right)$ may be represented as a concatenation $\eta_{1} \circ$ $\eta_{2} \circ \cdots \circ \eta_{N}$ of oriented arcs $\eta_{a}$, each contained within a three-holed sphere $C_{0,3}^{t}$. It will not cause a loss of generality to assume that each arc $\eta_{a}$ is of the following two types:

- An arc $[j i]_{t}$ on $C_{0,3}^{t}$ running from the marked point on boundary component $i$ of trinion (three punctured sphere) $t$ to the one on boundary component $j$ as depicted in Figure 1 ,

- An arc $b_{i}^{t}$ connecting the two marked points on boundary component $i$ of $C_{0,3}^{t}$ with positive orientation 5 .

We will assume that the point $y_{0}$ is located on the boundary circle $\delta_{n}$ of $C_{0, n}$. It will be useful to introduce the notation $\left[j i^{\nu}\right]_{t}$ for the composite $\operatorname{arcs}\left(b_{i}^{t}\right)^{\nu} \circ[j i]_{t}, \nu \in \mathbb{Z}$.

\subsubsection{The algorithm}

Using the results from Subsection 3.3 and the definitions from 3.4 .1 we may now formulate a simple algorithm for calculating the result of the analytic continuation $\mathcal{F}\left(\alpha ; \beta|z| y_{0} \mid y\right)$ along $\chi_{k}$. We will use the geometrical set-up introduced in Subsection 3.4.1, in particular the decomposition of the paths $\chi_{k}$ into a collection of arcs. Note that the basic building blocks are close relatives of the moves introduced in (3.31) such as

$$
\begin{aligned}
\psi_{s_{2}}(y) V_{\alpha_{3}, \alpha_{1}}^{\alpha_{2}}\left[v_{2}\right](z) & =\psi_{s_{2}}(y) V_{\alpha_{3}+s_{2} \frac{b}{2}, \alpha_{1}}^{\alpha_{2}}\left[v_{2}\right](z) \cdot \bigvee_{\alpha_{3}}^{\frac{1}{2} s_{2}} \\
& =\sum_{s_{1}= \pm 1} F_{-s_{2}, s_{1}}^{[23]} V_{\alpha_{3}, \alpha_{1}}^{\alpha_{2}-s_{1} \frac{b}{2}}\left[\psi_{s_{1}}(y-z) v_{2}\right](z) \cdot \mathrm{V}_{\alpha_{3}}^{\frac{1}{2} s_{2}}
\end{aligned}
$$

In this way we find that the arcs $\left[j i^{\nu}\right]_{t}$ are represented by the matrices

$$
\mathrm{S}_{[j i]}^{t}:=\mathrm{F}_{[j i]}^{t} \cdot \mathrm{T}_{i}^{t}, \quad \mathrm{C}_{[j i]}^{t, \nu}:=\mathrm{S}_{[j i]}^{t} \cdot\left(\mathrm{B}_{i}^{t}\right)^{\nu},
$$

where

\footnotetext{
${ }^{5}$ The orientation is indicated in Figure 1. The starting point is determined either by the base point or by the end-point of the previous arc.
} 
- $\mathrm{F}_{[j i]}^{t}$ is obtained from $F^{[j i]}$ by replacing $\alpha_{i} \rightarrow \alpha_{i}^{t}, i=1,2,3$ and transposition

- $\mathrm{T}_{i}^{t}$ is defined as

$$
\left(\mathrm{T}_{i}^{t}\right)_{s_{1} s_{2}}=\delta_{s_{1},-s_{2}}\left(\mathrm{~V}_{t}\right)^{\frac{1}{2} s_{2}}
$$

where $\mathrm{V}_{t}$ is the shift operator which shifts the variable $\alpha_{t} \equiv \alpha_{i}^{t}$ as defined in equation (3.37). The operators $V_{t}$ act to the left in the product of matrices.

- $\mathrm{B}_{i}^{t}$ is the matrix with elements

$$
\left(\mathrm{B}_{i}^{t}\right)_{s_{1} s_{2}}=\delta_{s_{1} s_{2}} B_{s_{1}}\left(\alpha_{i}\right)
$$

Arcs $b_{i}^{t}$ will be represented by the matrix $\mathrm{B}_{i}^{t}$. If $\chi_{k}$ is a simple closed curve on $C_{0, n}$ starting and ending at $y_{0}$ represented by the ordered concatenation $\eta_{1} \circ \eta_{2} \circ \cdots \circ \eta_{K}$ of the arcs defined above, we will define

$$
\mathrm{M}_{k}=\mathrm{N}_{K} \cdot \mathrm{N}_{K-1} \cdots \mathrm{N}_{1}
$$

where $\mathrm{N}_{k}$ are the $2 \times 2$-matrices associated to the arcs $\eta_{k}$. We may thereby define the sought-for collection of matrices $\mathrm{M}_{k}, k=1, \ldots, n$ describing the action of monodromies of the degenerate fields on spaces of conformal blocks.

One should not forget that the resulting monodromy matrix is operator-valued: it is a matrix which has elements containing the operators $\mathrm{V}_{t}$ shifting the parameters $\beta$.

\section{Solving the Riemann-Hilbert problem}

We shall now specialize to $c=1$. For that case we shall replace the parameters $\alpha_{k}$ and $\beta_{r}$ by variables $m_{k}$ and $p_{r}$ giving the conformal dimensions as $\Delta_{m_{k}}=m_{k}^{2}$ and $\Delta_{p_{r}}=p_{r}^{2}$, for $k=1, \ldots, n$ and $r=1, \ldots, n-3$, respectively.

\subsection{The construction}

Let us now consider,

$$
\begin{aligned}
& \mathcal{F}_{s^{\prime} s}\left(m ; p|z| y_{0} \mid y\right):=\left\langle m_{n}\left|\psi_{-s^{\prime}}\left(y_{0}\right) \psi_{s}(y)\right| \Theta_{s-s^{\prime}}\right\rangle, \\
& \left|\Theta_{\epsilon}\right\rangle=V_{m_{n}+\frac{\epsilon}{2}, p_{n-3}}^{m_{n-1}}\left(z_{n-1}\right) \ldots V_{p_{2}, p_{1}}^{m_{3}}\left(z_{3}\right) V_{p_{1}, m_{1}}^{m_{2}}\left(z_{2}\right)\left|m_{1}\right\rangle,
\end{aligned}
$$

${ }^{6}$ We are here representing fusion and braid moves by matrix multiplication from the right to be consistent with [2.1). This differs from the conventions used in [DGOT] where multiplication from the left was used. The matrices written below are therefore related to those of [DGOT] by transposition. 
where $V_{p_{2}, p_{1}}^{m}(z)$ maps $\mathcal{V}_{p_{1}}$ to $\mathcal{V}_{p_{2}}$ and $\psi_{s}(y)$ maps $\mathcal{V}_{p}$ to $\mathcal{V}_{p-s / 2}$ for all $p$. We will from now on assume that the vertex operators $V_{p_{2}, p_{1}}^{m}(z)$ are normalized by (3.18) with $N\left(p_{3}, p_{2}, p_{1}\right)$ being chosen as

$$
\begin{aligned}
& N\left(p_{3}, p_{2}, p_{1}\right)= \\
& =\frac{G\left(1+p_{3}-p_{2}-p_{1}\right) G\left(1+p_{1}-p_{3}-p_{2}\right) G\left(1+p_{2}-p_{1}-p_{3}\right) G\left(1+p_{3}+p_{2}+p_{1}\right)}{G\left(1+2 p_{3}\right) G\left(1-2 p_{2}\right) G\left(1-2 p_{1}\right)},
\end{aligned}
$$

where $G(p)$ is the Barnes $G$-function that satisfies $G(p+1)=\Gamma(p) G(p)$.

Consider the matrix $\Psi\left(y ; y_{0}\right)$ which has elements

$$
\Psi_{s^{\prime} s}\left(y ; y_{0}\right):=\frac{\pi s^{\prime}\left(y_{0}-y\right)^{\frac{1}{2}}}{\sin 2 \pi m_{n}} \frac{\left\langle m_{n}\left|\psi_{-s^{\prime}}\left(y_{0}\right) \psi_{s}(y)\right| \Theta_{s-s^{\prime}}^{\mathrm{D}}\right\rangle}{\left\langle m_{n} \mid \Theta_{0}^{\mathrm{D}}\right\rangle}
$$

where

$$
\left|\Theta_{\epsilon}^{\mathrm{D}}(\sigma, \tau)\right\rangle:=\sum_{\vec{n} \in \mathbb{Z}^{N}} \prod_{r=1}^{N} e^{i n_{r} \tau_{r}}\left|\Theta_{\epsilon}(\sigma+\vec{n})\right\rangle .
$$

We have introduced $N:=n-3$, and the summation is over vectors $\vec{n}=\left(n_{1}, \ldots, n_{N}\right)$ in $\mathbb{Z}^{N}$. We claim that $\Psi_{s^{\prime} s}\left(y ; y_{0}\right)$ represents the sought-for solution to the Riemann-Hilbert problem. The proof of this statement is given in the following subsections. At this point we only remark that the prefactor in 4.43 a) ensures the normalization $\Psi\left(y_{0} ; y_{0}\right)=1$.

The observations above provide the input needed to apply the reasoning presented in [GIL] to show that the isomonodromic tau-function is nothing but

$$
\tau(z)=\left\langle m_{n} \mid \Theta_{0}^{\mathrm{D}}\right\rangle
$$

Our results for the case $n=4$ yield in particular a proof of the relation between the tau function for Painlevé VI and Virasoro conformal blocks discovered in [GIL].

\subsection{Existence of classical monodromies}

We may calculate the monodromies by the algorithm formulated in Subsection 3.4.2 with input data $F_{s_{1}, s_{2}}^{[j i]}$ and $B_{s}(\alpha)$ now given by

$$
\begin{aligned}
& F_{s_{1}, s_{2}}^{[j i]}=s_{1} \frac{\cos \pi\left(p_{k}+s_{2} p_{j}-s_{1} p_{i}\right)}{\sin 2 \pi p_{j}}, \\
& B_{s}(p)=e^{-\pi \mathrm{i} s p} .
\end{aligned}
$$

The operator $\mathrm{V}_{t}$ may now be represented as $\mathrm{V}_{t}=e^{\mathrm{i} \mathrm{q}_{t}}$, where $\mathrm{q}_{t}=i \frac{\partial}{\partial p_{t}}$. Let us denote the resulting operator-valued monodromy matrices by $\mathrm{M}_{\gamma}$. 
We may now make a key observation: the monodromy matrices $\mathrm{M}_{\gamma}$ have matrix elements that are rational functions of $\mathrm{U}_{t}=e^{2 \pi i p_{t}}$ and $\mathrm{V}_{t}$ which generate a commutative subalgebra of the algebra of all operators 7 acting on the space of conformal blocks.

In order to see that $\mathrm{M}_{\gamma}$ depends only on $\mathrm{V}_{t}$ rather than $\left(\mathrm{V}_{t}\right)^{\frac{1}{2}}$ let us note that each curve of the cut system traversed on the way must be crossed a second time before one can return to the starting point. In a similar way one may see that $\mathrm{M}_{\gamma}$ depends on $p_{t}$ only via $U_{t}=e^{2 \pi i p_{t}}$ : the elements of the matrices $F_{s_{1}, s_{2}}^{[j i]}$ are linear combinations of the form $A e^{\pi i p_{t}}+B e^{-\pi i p_{t}}$. As the product of matrices representing $\mathrm{M}_{\gamma}$ will always contain an even number of matrices depending on a given variable $p_{t}$, it follows that $\mathrm{M}_{\gamma}$ depends on $p_{t}$ only via $e^{2 \pi i p_{t}}$.

But this means that the algebra generated by the matrix elements of $M_{\gamma}$ becomes classical (commutative) in the limit $c \rightarrow 1$ ! This allows us to diagonalize the operator $\mathrm{V}_{t}$ by taking linear combinations of the form (4.43b). The generalized Fourier-transformation (4.43b) diagonalizes $\mathrm{V}_{t}$ with eigenvalue $e^{i \tau_{t}}$, while $e^{2 \pi i p_{t}}$ will act on $\Psi_{s^{\prime} s}$ by multiplication. The matrix obtained from $\mathrm{M}_{\gamma}$ by means of the transformation (4.43b) will be denoted $M_{\gamma}$.

\subsection{Calculation of monodromies}

In order to formulate the rules for the calculation of the monodromy matrices $M_{k}$, let us assume without loss of generality that the path connecting boundary component $\delta_{n}$ to $\delta_{k}$ passes through the trinions $t_{1}, t_{2}, \ldots, t_{L}$ in the given order, each trinion being traversed exactly once. We claim that we may then calculate the monodromy matrices $M_{k}$ as

$$
M_{k}=\sigma_{3} \cdot\left[C_{\left[j_{L} i_{L}\right]}^{t_{L}, \nu_{L}} \ldots C_{\left[j_{1} i_{1}\right]}^{t_{1}, \nu_{1}}\right]^{-1} \cdot\left(\mathrm{B}_{k}^{t_{L}}\right)^{2} \cdot\left[C_{\left[j_{L} i_{L}\right]}^{t_{L}, \nu_{L}} \ldots C_{\left[j_{1} i_{1}\right]}^{t_{1}, \nu_{1}}\right] \cdot \sigma_{3},
$$

where $\sigma_{3}=\left(\begin{array}{rr}1 & 0 \\ 0 & -1\end{array}\right)$ and $C_{[j i]}^{t, \nu}$ is defined as

$$
C_{[j i]}^{t, \nu}:=F_{[j i]}^{t} \cdot(T B)_{i}^{t, \nu},
$$

with matrices $F_{[j i]}^{t}$, and $(T B)_{i}^{t, \nu}$ defined as

$$
\begin{aligned}
& \left(F_{[j i]}^{t}\right)_{s_{1}, s_{2}}=s_{2} \frac{\sin \pi\left(p_{k}^{t}+s_{2} p_{j}^{t}-s_{1} p_{i}^{t}\right)}{\sin 2 \pi p_{j}^{t}}, \\
& {\left[(T B)_{i}^{t, \nu}\right]_{s_{1} s_{2}}=\delta_{s_{1},-s_{2}} \mathrm{i}^{s_{2} \nu} e^{s_{2} \frac{\mathrm{i}}{2} \tau_{i}^{t}} e^{-\pi \mathrm{i} \nu s_{2} p_{i}^{t}} .}
\end{aligned}
$$

In order to derive these rules let us note that application of the algorithm formulated in Subsection 3.4.2 will produce monodromy matrices $\mathrm{M}_{k}$ of the following form:

$$
\mathrm{M}_{k}=\left[\mathrm{C}_{\left[j_{L} i_{L}\right]}^{t_{L}, \nu_{L}} \cdot \ldots \mathrm{C}_{\left[j_{1} i_{1}\right]}^{t_{1}, \nu_{1}}\right]^{-1} \cdot\left(\mathrm{B}_{k}^{t_{L}}\right)^{2} \cdot\left[\mathrm{C}_{\left[j_{L} i_{L}\right]}^{t_{L}, \nu_{L}} \cdot \ldots \mathrm{C}_{\left[j_{1} i_{1}\right]}^{t_{1}, \nu_{1}}\right]
$$

\footnotetext{
${ }^{7}$ We mean operators acting on the conformal blocks built from $p_{t}, \partial_{p_{t}}$.
} 
The matrices $\mathrm{C}_{[j i]}^{t, \nu}, \nu \in \mathbb{Z}$, represent the contribution of the segments connecting boundary component $i$ to $j$ in trinion $t$. Recall that $\mathrm{C}_{[j i]}^{t, \nu}=\mathrm{S}_{[j i]}^{t} \cdot\left(\mathrm{B}_{i}^{t}\right)^{\nu}$. The matrices $\mathrm{S}_{[j i]}^{t}$ and $\left(\mathrm{S}_{[j i]}^{t}\right)^{-1}$ are explicitly given as

$$
\begin{aligned}
& \mathrm{S}_{[j i]}^{t}=\frac{1}{\sin 2 \pi p_{j}^{t}}\left(\begin{array}{ll}
-\sin \pi\left(p_{k}^{t}+p_{j}^{t}-p_{i}^{t}-\frac{1}{2}\right) & \sin \pi\left(p_{k}^{t}-p_{j}^{t}-p_{i}^{t}+\frac{1}{2}\right) \\
-\sin \pi\left(p_{k}^{t}+p_{j}^{t}+p_{i}^{t}-\frac{3}{2}\right) & \sin \pi\left(p_{k}^{t}-p_{j}^{t}+p_{i}^{t}-\frac{1}{2}\right)
\end{array}\right)\left(\begin{array}{cc}
0 & e^{-\frac{i}{2} \mathrm{q}_{i}^{t}} \\
e^{\frac{i}{2} \mathrm{q}_{i}^{t}} & 0
\end{array}\right) \\
& =\frac{1}{\sin 2 \pi p_{j}^{t}}\left(\begin{array}{ll}
-e^{\frac{i}{2} q_{i}^{t}} \sin \pi\left(p_{k}^{t}-p_{j}^{t}-p_{i}^{t}\right) & e^{-\frac{i}{2} q_{i}^{t}} \sin \pi\left(p_{k}^{t}+p_{j}^{t}-p_{i}^{t}\right) \\
-e^{\frac{i}{2} q_{i}^{t}} \sin \pi\left(p_{k}^{t}-p_{j}^{t}+p_{i}^{t}\right) & e^{-\frac{i}{2} q_{i}^{t}} \sin \pi\left(p_{k}^{t}+p_{j}^{t}+p_{i}^{t}\right)
\end{array}\right), \\
& \left(\mathrm{S}_{[j i]}^{t}\right)^{-1}=\frac{1}{\sin 2 \pi p_{i}^{t}}\left(\begin{array}{ll}
\sin \pi\left(p_{k}^{t}+p_{j}^{t}+p_{i}^{t}\right) e^{-\frac{\mathrm{i}}{2} \mathbf{q}_{i}^{t}} & -\sin \pi\left(p_{k}^{t}+p_{j}^{t}-p_{i}^{t}\right) e^{-\frac{\mathrm{i}}{2} \mathbf{q}_{i}^{t}} \\
\sin \pi\left(p_{k}^{t}-p_{j}^{t}+p_{i}^{t}\right) e^{+\frac{\mathrm{i}}{2} q_{i}^{t}} & -\sin \pi\left(p_{k}^{t}-p_{j}^{t}-p_{i}^{t}\right) e^{+\frac{\mathrm{i}}{2} \mathrm{q}_{i}^{t}}
\end{array}\right) .
\end{aligned}
$$

In order to calculate the effect of the transformation (4.43b) it is convenient to move the operators $e^{ \pm \mathrm{iq} t}$ to the left in (4.48). To this aim let us analyze the dependence of $\mathrm{M}_{k}$ on $p_{i_{a}} \equiv p_{i_{a}}^{t_{a}}$ and the shift operator $e^{\mathrm{i} q_{a}}$, where $\mathrm{q}_{a} \equiv \mathrm{q}_{i_{a}}^{t_{a}}$. The dependence on $e^{\mathrm{i} \mathrm{q}_{a}}$ can be made explicit by writing $\mathrm{M}_{k}$ as

$$
\mathrm{M}_{k}=\left[\mathrm{C}^{t_{a-2} \ldots t_{1}}\right]^{-1} \cdot\left[\mathrm{C}_{\left[j_{a-1} i_{a-1}\right]}^{t_{a-1} \nu_{a-1}}\right]^{-1} \cdot\left[\mathrm{B}_{i_{a}}^{t_{a}}\right]^{-\nu_{a}} \cdot \mathrm{M}_{k, a}^{\prime} \cdot\left(\mathrm{B}_{i_{a}}^{t_{a}}\right)^{\nu_{a}} \cdot \mathrm{C}_{\left[j_{a-1} i_{a-1}\right]}^{t_{a-1}, \nu_{a-1}} \cdot\left[\mathrm{C}^{t_{a-2} \ldots t_{1}}\right],
$$

where

$$
\mathrm{M}_{k, a}^{\prime}:=\left[\mathrm{S}_{\left[j_{a} i_{a}\right]}^{\left.t_{a}\right]}\right]^{-1} \cdot\left[\mathrm{C}_{\left[j_{L} i_{L}\right]}^{t_{L}, \nu_{L}} \cdots \mathrm{C}_{\left[j_{a+1} i_{a+1}\right]}^{t_{a+1}, \nu_{a+1}}\right]^{-1} \cdot\left(\mathrm{B}_{k}^{t_{L}}\right)^{2} \cdot\left[\mathrm{C}_{\left[j_{L} i_{L}\right]}^{t_{L}, \nu_{L}} \cdots \mathrm{C}_{\left[j_{a+1} i_{a+1}\right]}^{\left.t_{a+1}, \nu_{a+1}\right]}\right] \mathrm{S}_{\left[j_{a} i_{a}\right]}^{t_{a}} \cdot
$$

It is easy to see that the dependence of the matrix $\mathrm{M}_{k, a}^{\prime}$ on $e^{\mathrm{i} q_{a}}$ is of the form

$$
\mathrm{M}_{k, a}^{\prime}=\left(\begin{array}{rr}
\left(\mathrm{m}_{k, a}^{\prime}\right)_{++} & -e^{-\mathrm{i} \mathrm{q}_{a}}\left(\mathrm{~m}_{k, a}^{\prime}\right)_{+-} \\
-e^{\mathrm{i} \mathrm{q}_{a}}\left(\mathrm{~m}_{k, a}^{\prime}\right)_{-+} & \left(\mathrm{m}_{k, a}^{\prime}\right)_{--}
\end{array}\right),
$$

where $\mathrm{m}_{k, a}^{\prime}$ is the matrix one would obtain by replacing $\mathrm{q}_{a}$ by 0 and $\mathrm{F}_{\left[j_{a} i_{a}\right]}^{t_{a}}$ by $F_{\left[j_{a} i_{a}\right]}^{t_{a}}$ from the very beginning. The extra minus sign is the result of the application of the exchange relation

$$
\sin \pi\left(p_{k_{a}}+p_{j_{a}}+p_{i_{a}}\right) e^{-\mathrm{i} \mathrm{q}_{a}}=-e^{-\mathrm{i} q_{a}} \sin \pi\left(p_{k_{a}}+p_{j_{a}}+p_{i_{a}}\right) .
$$

The only matrices in (4.49) to the left of $\mathrm{M}_{k, a}^{\prime}$ containing dependence on the variable $p_{i_{a}}$ are $\left[\mathrm{C}_{\left[j_{a-1} i_{a-1}\right]}^{t_{a}, \nu_{a-1}}\right]^{-1}$ and $\left[\mathrm{B}_{i_{a}}^{t_{a}}\right]^{-\nu_{a}}$. The matrix elements of both $\mathrm{S}_{\left[j_{a-1} i_{a-1}\right]}^{t_{a}}$ and $\mathrm{B}_{i_{a}}^{t_{a}}$ are both anti-periodic under shifts $p_{a} \rightarrow p_{a}+1$. Moving $e^{ \pm \mathrm{i} q_{a}}$ through the product $\left[\mathrm{C}_{\left[j_{a-1} i_{a-1}\right]}^{t_{a-1}, \nu_{a-1}}\right]^{-1} \cdot\left[\mathrm{B}_{i_{a}}^{t_{a}}\right]^{-\nu_{a}}$ will for $a>1$ produce an extra sign $(-)^{1+\nu_{a}}$. This sign is taken into account by means of the factor $\mathrm{i}^{s_{2} \nu_{a}}$ in (4.47c). The extra sign $(-)^{1+\nu_{a}}$ should be replaced by $(-)^{\nu_{a}}$ in the case $a=1$. This is taken into account by means of conjugation with $\sigma_{3}$ in (4.46).

Calculating the trace functions $L_{s}^{r}$ and $L_{t}^{r}$ using the algorithm above shows that the parameters $(\sigma, \tau)$ coincide with those introduced in Subsection 2.2. The details are given in Appendix $\mathrm{A}$ 


\section{Non-Abelian fermionization}

It was shown in the work of Sato, Jimbo and Miwa that the isomonodromic tau-functions can be represented in terms of free fermion correlators. Our results give a "bosonic" representation for the isomonodromic tau-functions in terms of Virasoro vertex operators. In this section we will clarify the relation between these two constructions by showing that our construction is essentially equivalent to a bosonic construction of twist fields creating singularities with nontrivial monodromy. It seems natural to regard our construction as the bosonization of the fermionic construction of twist fields presented by Sato, Jimbo and Miwa.

\subsection{Fermions from degenerate fields}

Let us introduce a free field $\varphi_{0}$,

$$
\varphi_{0}(w) \varphi_{0}(z) \sim-\frac{1}{2} \log (w-z) .
$$

Note furthermore that we have

$$
\left.\Delta_{-b / 2}\right|_{b=i}=\frac{1}{4},\left.\quad \Delta_{-b}\right|_{b=i}=1 .
$$

Construct the fields

$$
\Psi_{s}(z):=e^{i \varphi_{0}(z)} \psi_{s}(z), \quad \bar{\Psi}_{s}(z):=e^{-i \varphi_{0}(z)} \psi_{-s}(z) .
$$

These fields have the OPE

$$
\begin{aligned}
& \Psi_{s}(w) \Psi_{s^{\prime}}(z) \sim \text { regular }, \\
& \Psi_{s}(w) \bar{\Psi}_{s^{\prime}}(z) \sim \frac{\delta_{s, s^{\prime}}}{w-z} .
\end{aligned}
$$

This means that the fields $\Psi_{s}(w), \bar{\Psi}_{s}(w)$ generate a representation of the fermionic vertex operator algebra $\mathfrak{F}$. The action of these fields can be restricted to the spaces $\mathcal{F}_{\sigma, \tau}$, defined as

$$
\mathcal{F}_{\sigma, \tau}:=\bigoplus_{\substack{k, l \in \frac{1}{2} \mathbb{Z} \\ k+l \in \mathbb{Z}}} \mathcal{F}_{\sigma, \tau}^{[k, l]}, \quad \mathcal{F}_{\sigma, \tau}^{[k, l]}:=\mathcal{V}_{\sigma-k} \otimes \mathcal{F}_{\tau+l}
$$

with $\mathcal{F}_{\tau}$ being the free boson Fock space with eigenvalue $\tau$ for the zero mode of $\partial \varphi_{0}$. Note that the action of $\Psi_{s}(z), \bar{\Psi}_{s}(z)$ shifts $k+l$ by an integer amount. In order to get a label for inequivalent representations of (5.52) we may restrict $\sigma$ and $\tau$ to $0 \leq \Re(\sigma)<1 / 2$ and $0 \leq \Re(\tau)<1$, respectively.

The restriction of $\Psi_{s}(z), \bar{\Psi}_{s}(z)$ to $\mathcal{F}_{\sigma, \tau}$ has monodromy

$$
\Psi_{s}\left(e^{2 \pi i} z\right)=e^{2 \pi i(\tau-s \sigma)} \Psi_{s}(z), \quad \bar{\Psi}_{s}\left(e^{2 \pi i} z\right)=e^{-2 \pi i(\tau-s \sigma)} \bar{\Psi}_{s}(z) .
$$


Other representations of the fermionic vertex operator algebra (5.52) can be defined by taking linear combinations

$$
\Phi_{s}(z):=\sum_{t= \pm} C_{s t} \Psi_{t}(z), \quad \bar{\Phi}_{s}(z):=\sum_{t= \pm}\left(C^{-1}\right)_{s t} \bar{\Psi}_{t}(z)
$$

for any element $C$ of $\mathrm{GL}(2)$. The representation is characterized by the $\mathrm{GL}(2)$-monodromy

$$
\begin{array}{ll}
\Phi_{s}\left(e^{2 \pi i} z\right)=\sum_{t= \pm} M_{s t} \Phi_{t}(z), & \bar{\Phi}_{s}\left(e^{2 \pi i} z\right):=\sum_{t= \pm}\left(M^{-1}\right)_{s t} \bar{\Phi}_{t}(z), \\
\text { where } M=C \cdot e^{2 \pi i D} \cdot C^{-1}, & D:=\operatorname{diag}(\tau-\sigma, \tau+\sigma) .
\end{array}
$$

It seems natural to consider equivalence classes of representations defined by identifying representations related by the similarity transformation (5.55). Slightly abusing notations we will denote the representations characterized by monodromy of the form $(5.56)$ by $\mathcal{F}_{\sigma, \tau}$.

It will be useful to decompose $\mathcal{F}_{\sigma, \tau}$ as

$$
\mathcal{F}_{\sigma, \tau}=\mathcal{F}_{\sigma, \tau}^{0} \oplus \mathcal{F}_{\sigma, \tau}^{1 / 2}, \quad \text { where } \quad \mathcal{F}_{\sigma, \tau}^{\epsilon}:=\bigoplus_{\substack{k \in \mathbb{Z}, l \in \frac{1}{2} \mathbb{Z} \\ k+l \in \mathbb{Z}}} \mathcal{F}_{\sigma, \tau}^{[k+\epsilon, l]},
$$

assuming that $\epsilon \in \frac{1}{2} \mathbb{Z}_{2}$. The action of a field $\Psi_{s}(w), \bar{\Psi}_{s}(w)$ maps $\mathcal{F}_{\sigma, \tau}^{0}$ to $\mathcal{F}_{\sigma, \tau}^{1 / 2}$ and vice-versa.

\subsection{Chiral vertex operators for free fermion representations}

Let us then define the vertex operators

$$
\Phi_{\sigma_{3}, \tau_{3} ; \sigma_{1}, \tau_{1}}^{\sigma_{2}, \epsilon_{2}, q_{3}}(z): \mathcal{F}_{\sigma_{1}, \tau_{1}} \rightarrow \mathcal{F}_{\sigma_{3}, \tau_{3}}
$$

by defining their action on arbitrary vectors $v_{1} \in \mathcal{F}_{\sigma_{1}, \tau_{1}}^{\left[k_{1}+\epsilon_{1}, l_{1}\right]}$ to be

$$
\Phi_{\sigma_{3}, \tau_{3} ; \sigma_{1}, \tau_{1}}^{\sigma_{2}, \tau_{2} ; \epsilon_{2}, q_{3}}(z) v_{1}:=e^{2 i \tau_{2} \varphi_{0}(z)} \sum_{n \in \mathbb{Z}} e^{i n q_{3}} V_{\sigma_{3}-\left[\epsilon_{1}+\epsilon_{2}\right]+n ; \sigma_{1}-k_{1}-\epsilon_{1}}^{\sigma_{2}-\epsilon_{2}}(z) v_{1}
$$

we assume that $\tau_{3}=\tau_{2}+\tau_{1}$, and define $[\epsilon]=0$ if $\epsilon \in \mathbb{Z},[\epsilon]=1 / 2$ if $\epsilon \in \mathbb{Z}+\frac{1}{2}$. The definition is such that the restriction of $\Phi_{\sigma_{3}, \tau_{3} ; \sigma_{1}, \tau_{1}, \tau_{1}}^{\sigma_{2}, \tau_{3}}(z)$ to the subspace $\mathcal{F}_{\sigma_{1}, \tau_{1}}^{\epsilon_{1}}$ of $\mathcal{F}_{\sigma_{1}, \tau_{1}}$ yields an operator with image contained in the subspace $\mathcal{F}_{\sigma_{3}, \tau_{3}}^{\epsilon_{1}+\epsilon_{2}}$ of $\mathcal{F}_{\sigma_{3}, \tau_{3}}$. This selection rule expresses conservation of the quantum number $\epsilon \in \frac{1}{2} \mathbb{Z}_{2}$.

The relations (3.31c) combined with the standard braid relations of normal ordered exponentials imply the following exchange relations between the vertex operators $\Phi_{\sigma_{3}, \tau_{3}}^{\sigma_{2}, \tau_{2} ; \sigma_{1}, \tau_{1}}(z)$ and the fermion fields $\Psi_{s}(w)$,

$$
\begin{aligned}
& \Psi_{s}(w \pm i 0) \Phi_{\sigma_{3}, \tau_{3}}^{\sigma_{2}, \tau_{2} ; \sigma_{1}, \tau_{1}, q_{3}}(z)=\Phi_{\sigma_{3}, \tau_{3} ; \sigma_{1}, \tau_{1}}^{\sigma_{2}, \tau_{2} ; \epsilon_{2}, q_{3}}(z) \sum_{t= \pm} \Psi_{t}(w \pm i 0) B^{ \pm}\left(q_{3}\right)_{t, s} \\
& \bar{\Psi}_{s}(w \pm i 0) \Phi_{\sigma_{3}, \tau_{3} ; \sigma_{1}, \tau_{1}}^{\sigma_{2}, \tau_{2} ; \epsilon_{2}, q_{3}}(z)=\Phi_{\sigma_{3}, \tau_{3} ; \sigma_{1}, \tau_{1}}^{\sigma_{2}, \tau_{2} ; \epsilon_{2}, q_{3}}(z) \sum_{t= \pm} \bar{\Psi}_{t}(w \pm i 0) \bar{B}^{ \pm}\left(q_{3}\right)_{t, s}
\end{aligned}
$$


The matrices $B_{t, s}^{ \pm}\left(q_{3}\right)$ and $\bar{B}_{t, s}^{ \pm}\left(q_{3}\right)$ are explicitly given as

$$
\begin{aligned}
& B^{ \pm}\left(q_{3}\right)_{t, s}=e^{ \pm \pi i \tau_{2}} e^{\mp \pi i\left(s \sigma_{3}-t \sigma_{1}\right)} e^{i\left(\left[\epsilon_{3}+\frac{1}{2}\right]-\epsilon_{3}-\frac{s}{2}\right) q_{3}} F_{s t}^{[13]}\left(\sigma_{3}, \sigma_{2}-\epsilon_{2}, \sigma_{1}\right), \\
& \bar{B}^{ \pm}\left(q_{3}\right)_{t, s}=e^{\mp \pi i \tau_{2}} e^{ \pm \pi i\left(s \sigma_{3}-t \sigma_{1}\right)} e^{i\left(\left[\epsilon_{3}+\frac{1}{2}\right]-\epsilon_{3}+\frac{s}{2}\right) q_{3}} F_{-s,-t}^{[13]}\left(\sigma_{3}, \sigma_{2}-\epsilon_{2}, \sigma_{1}\right) .
\end{aligned}
$$

The exchange relations (5.60) express the fact that $\left.\Phi_{\sigma_{3}, \tau_{3} ; \sigma_{2}, \tau_{1}}^{\sigma_{2} ;} ; z\right)$ is an intertwiner between the representations $\mathcal{F}_{\sigma_{1}, \tau_{1}}$ and $\mathcal{F}_{\sigma_{3}, \tau_{3}}$ of the free fermion algebra $\mathfrak{F}$. It also follows from these observations that the vertex operators $\Phi_{\sigma_{3}, \tau_{3} ; \sigma_{1}, \tau_{1}}^{\sigma_{2}, \tau_{2} ; \epsilon_{2}, q_{3}}(z)$ represent twist fields: They create states in which the fermions $\Psi_{s}(z)$ have monodromy $B^{-}\left(q_{3}\right)\left(B^{+}\left(q_{3}\right)\right)^{-1}$ around $z$.

An important consequence of (5.60) is the fact that matrix elements of compositions of the vertex operators $\left.\Phi_{\sigma_{3}, \tau_{3}}^{\sigma_{2}, \tau_{2} ; \sigma_{1}, \tau_{1}}, \tau_{2}\right)$ such as

$$
\left\langle e_{\sigma_{4}, \tau_{4}}^{\epsilon_{4}}\left|\Phi_{\sigma_{4}, \tau_{4} ; \sigma, \tau}^{\sigma_{3}, \tau_{3} ; \epsilon_{3}, q_{4}}\left(z_{3}\right) \Phi_{\sigma, \tau}^{\sigma_{2}, \tau_{2} ; \epsilon_{2}, q_{3}} ; \sigma_{1}, \tau_{1}\left(z_{2}\right)\right| e_{\sigma_{1}, \tau_{1}}^{\epsilon_{1}}\right\rangle
$$

represent conformal blocks for the free fermion algebra $\mathfrak{F} .\left|e_{\sigma, \tau}^{\epsilon}\right\rangle$ is the product of highest weight vertors in $\mathcal{V}_{\sigma-\epsilon} \otimes \mathcal{F}_{\tau}$. It follows from the conservation of the quantum number $\epsilon$ that such conformal blocks are non-vanishing only if $\epsilon_{4}=\epsilon_{1}+\epsilon_{2}+\epsilon_{3} \bmod 1$. Conservation of the zero mode of $\varphi_{0}$ implies furthermore that $\tau_{4}=\tau_{1}+\tau_{2}+\tau_{3}$.

The free fermion conformal blocks factorize as

$$
\begin{aligned}
\left\langle e_{\sigma_{4}, \tau_{4}}^{\epsilon_{4}}\left|\Phi_{\sigma_{4}, \tau_{4}}^{\sigma_{3}, \tau_{2} ; \epsilon_{3}, q_{4}}\left(z_{3}\right) \Phi_{\sigma, \tau ; \sigma_{1}, \tau_{1}}^{\sigma_{2}, \tau_{2} ; \epsilon_{2}, q_{3}}\left(z_{2}\right)\right| e_{\sigma_{1}, \tau_{1}}^{\epsilon_{1}}\right\rangle_{\mathrm{FF}}= \\
=\left\langle\tau_{4}\left|e^{2 i \tau_{3} \varphi_{0}\left(z_{3}\right)} e^{2 i \tau_{2} \varphi_{0}\left(z_{2}\right)}\right| \tau_{1}\right\rangle_{0} \\
\quad \times \sum_{n \in \mathbb{Z}} e^{i n q}\left\langle\sigma_{4}-\epsilon_{4}\left|V_{\sigma_{4}-\epsilon_{4}, \sigma-\left[\epsilon_{1}+\epsilon_{2}\right]+n}^{\sigma_{3}-\epsilon_{3}}\left(z_{3}\right) V_{\sigma-\left[\epsilon_{1}+\epsilon_{2}\right]+n ; \sigma_{1}-\epsilon_{1}}^{\sigma_{2}-\epsilon_{2}}\left(z_{2}\right)\right| \sigma_{1}-\epsilon_{1}\right\rangle_{\text {Liou }}
\end{aligned}
$$

The factor in the last line was previously identified as the tau-function associated to isomonodromic deformations of SL(2)-connections, the free-field conformal block in the second line is nothing but the multiplier needed to get the tau-functions associated to the GL(2)-connections.

\section{Examples}

We now look at some of the applications of the above general formalism to the theory of monodromy preserving deformations. We start by providing a CFT derivation of the Jimbo's asymptotic formula [Ji] for the tau function of Painlevé VI equation. Next we show how the known algebro-geometric solutions of the Schlesinger system on $C_{0, n}[\mathrm{KK}]$ arise from conformal blocks of the Ashkin-Teller critical model [Za, ZZ]. 


\subsection{Painlevé VI and Jimbo's formula}

Consider the simplest nontrivial case of four punctures. The fundamental group $\pi_{1}\left(C_{0,4}\right)$ is isomorphic to free group of rank 3. Let $\chi_{1}, \ldots, \chi_{4}$ be the four loops shown in Figure $2 \mathrm{a}$, then

$$
\pi_{1}\left(C_{0,4}\right)=\left\langle\chi_{1}, \chi_{2}, \chi_{3}, \chi_{4} \mid \chi_{1} \circ \chi_{2} \circ \chi_{3} \circ \chi_{4}=1\right\rangle
$$

We denote by $M_{1}, \ldots, M_{4} \in \mathrm{SL}(2, \mathbb{C})$ the monodromy matrices associated to these loops, satisfying $M_{4} M_{3} M_{2} M_{1}=1$. Conjugacy classes of irreducible representations of $\pi_{1}\left(C_{0,4}\right)$ in $\mathrm{SL}(2, \mathbb{C})$ are uniquely specified by seven invariants

$$
\begin{gathered}
L_{k}=\operatorname{Tr} M_{k}=2 \cos 2 \pi m_{k}, \quad k=1, \ldots, 4, \\
L_{s}=\operatorname{Tr} M_{1} M_{2}, \quad L_{t}=\operatorname{Tr} M_{2} M_{3}, \quad L_{u}=\operatorname{Tr} M_{1} M_{3},
\end{gathered}
$$

generating the algebra of invariant polynomial functions on $\operatorname{Hom}\left(\pi_{1}\left(C_{0,4}\right), \operatorname{SL}(2, \mathbb{C})\right)$. These traces satisfy the quartic equation

$$
\begin{aligned}
& L_{1} L_{2} L_{3} L_{4}+L_{s} L_{t} L_{u}+L_{s}^{2}+L_{t}^{2}+L_{u}^{2}+L_{1}^{2}+L_{2}^{2}+L_{3}^{2}+L_{4}^{2}= \\
& \quad=\left(L_{1} L_{2}+L_{3} L_{4}\right) L_{s}+\left(L_{2} L_{3}+L_{1} L_{4}\right) L_{t}+\left(L_{1} L_{3}+L_{2} L_{4}\right) L_{u}+4 .
\end{aligned}
$$

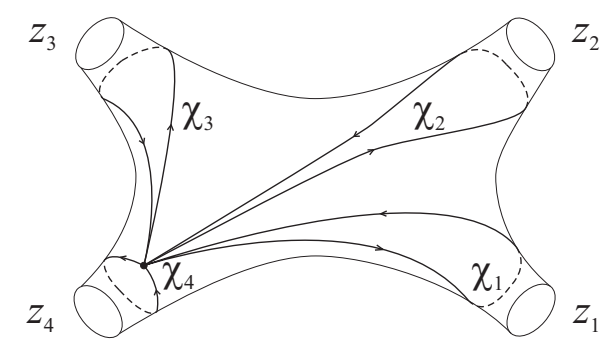

a)

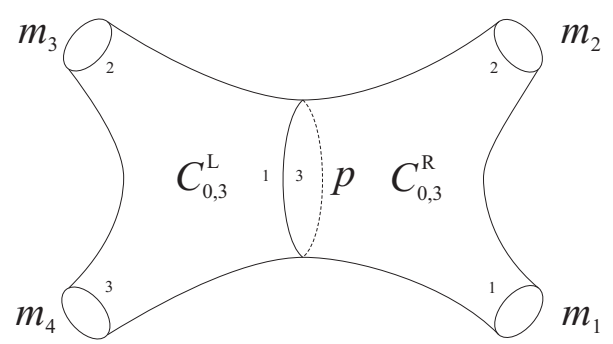

b)

Figure 2: Basis of loops of $\pi_{1}\left(C_{0,4}\right)$ and the decomposition $C_{0,4}=C_{0,3}^{L} \cup C_{0,3}^{R}$.

The affine algebraic variety defined by $(6.66)$ is the character variety of $C_{0,4}$. For every choice of $m_{1}, \ldots, m_{4}$, it defines a cubic surface in $\mathbb{C}^{3}$ in the variables $L_{s}, L_{t}, L_{u}$. If we further fix the trace function $L_{s}=2 \cos 2 \pi \sigma$, the resulting quadric in $L_{t}, L_{u}$ admits rational parameterization [Ji]

$$
\begin{aligned}
& \left(L_{s}^{2}-4\right) L_{t}=D_{t,+} \mathrm{s}+D_{t,-} \mathrm{s}^{-1}+D_{t, 0} \\
& \left(L_{s}^{2}-4\right) L_{u}=D_{u,+} \mathrm{s}+D_{u,-} \mathrm{s}^{-1}+D_{u, 0} .
\end{aligned}
$$


with coefficients given by

$$
\begin{aligned}
& D_{t, 0}=L_{s}\left(L_{1} L_{3}+L_{2} L_{4}\right)-2\left(L_{1} L_{4}+L_{2} L_{3}\right), \\
& D_{u, 0}=L_{s}\left(L_{2} L_{3}+L_{1} L_{4}\right)-2\left(L_{1} L_{3}+L_{2} L_{4}\right), \\
& D_{t, \pm}=16 \prod_{\epsilon= \pm} \sin \pi\left(m_{2} \mp \sigma+\epsilon m_{1}\right) \sin \pi\left(m_{3} \mp \sigma+\epsilon m_{4}\right), \\
& D_{u, \pm}=-D_{t, \pm} e^{\mp 2 \pi i \sigma} .
\end{aligned}
$$

The local coordinates $(\sigma, \mathrm{s})$ parameterize the space of $\mathrm{SL}(2, \mathbb{C})$-representations of $\pi_{1}\left(C_{0,4}\right)$ with fixed local monodromy exponents $m_{1}, \ldots, m_{4}$. Let us connect this pair to the parameters used in the conformal block representation of the fundamental matrix $Y(y)$.

The Riemann surface $C_{0,4}$ is glued from two three-holed spheres $C_{0,3}^{L}, C_{0,3}^{R}$ as shown in Figure $2 \mathrm{~b}$. The local coordinates $(p, \tau)$ associated to this pants decomposition parameterize trace functions via (2.5)-(2.6) (as well as their counterparts for $L_{u}$ ). Comparing these expressions with (6.67)-6.68), we find that

$$
\sigma=p, \quad \mathrm{~s}=\frac{\sin \pi\left(\sigma-m_{1}+m_{2}\right) \sin \pi\left(\sigma+m_{3}-m_{4}\right)}{\sin \pi\left(\sigma-m_{1}-m_{2}\right) \sin \pi\left(\sigma-m_{3}-m_{4}\right)} e^{i \tau} .
$$

Going back to the Schlesinger equations (2.10), note that three regular singularities $z_{1}, z_{3}, z_{4}$ can be brought to 0,1 and $\infty$ using Möbius transformations. The Schlesinger system then reduces to Painlevé VI equation

$$
\begin{aligned}
& -\frac{1}{2}\left(z(z-1) \zeta^{\prime \prime}\right)^{2}= \\
& =\operatorname{det}\left(\begin{array}{ccc}
2 m_{1}^{2} & z \zeta^{\prime}-\zeta & \zeta^{\prime}+m_{1}^{2}+m_{2}^{2}+m_{3}^{2}-m_{4}^{2} \\
z \zeta^{\prime}-\zeta & 2 m_{2}^{2} & (z-1) \zeta^{\prime}-\zeta \\
\zeta^{\prime}+m_{1}^{2}+m_{2}^{2}+m_{3}^{2}-m_{4}^{2} & (z-1) \zeta^{\prime}-\zeta & 2 m_{3}^{2}
\end{array}\right),
\end{aligned}
$$

satisfied by the logarithmic derivative of the tau function

$$
\zeta(z)=z(z-1) \frac{d}{d z} \ln \tau .
$$

Here $z=\frac{\left(z_{2}-z_{1}\right)\left(z_{4}-z_{3}\right)}{\left(z_{3}-z_{1}\right)\left(z_{4}-z_{2}\right)}$ denotes the cross-ratio of the singular points.

In the case of $C_{0,4}$, the representation (4.44) of $\tau(z)$ as a Fourier transform of the $c=1$ Virasoro conformal block is more explicitly written as

$$
\tau(z)=\sum_{n \in \mathbb{Z}}\left\langle m_{4}\left|V_{m_{4}, p+n}^{m_{3}}(1) V_{p+n, m_{1}}^{m_{2}}(z)\right| m_{1}\right\rangle e^{i n \tau} .
$$




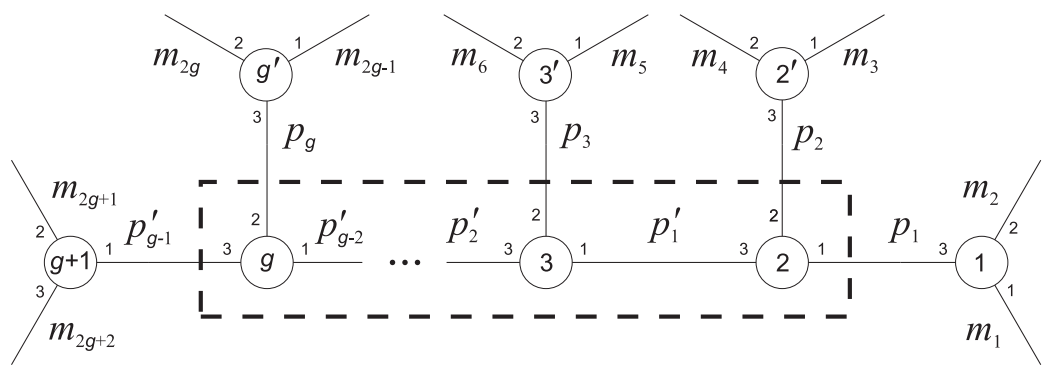

Figure 3: Labeling of pairs of pants for the conformal block $\mathcal{B}\left(m\left|p, p^{\prime}\right| z\right)$.

Assuming without loss of generality that $-\frac{1}{2}<\Re p<\frac{1}{2}$, letting $z \rightarrow 0$ in the last formula, and taking into account the normalization (4.42) of the chiral vertex operators, we deduce the asymptotics

$$
\begin{aligned}
\tau(z)= & \sum_{n=0, \pm 1} N\left(m_{4}, m_{3}, p+n\right) N\left(p+n, m_{2}, m_{1}\right) e^{i n \tau} z^{(p+n)^{2}-m_{1}^{2}-m_{2}^{2}}+ \\
& +O\left(z^{p^{2}-m_{1}^{2}-m_{2}^{2}+1}\right) .
\end{aligned}
$$

This is equivalent to the famous Jimbo's asymptotic formula [Ji, Theorem 1.1] expressing the critical behavior of the Painlevé VI tau function in terms of monodromy data. The relation of Jimbo parameters to ours is given by (6.69).

\subsection{Algebro-geometric solutions of the Schlesinger system}

Consider the pants decomposition of $C_{0,2 g+2}$ schematically depicted in Figure 3 , and denote by $\mathcal{B}\left(m\left|p, p^{\prime}\right| z\right)$ the corresponding $c=1$ conformal block. Its external legs are combined into $g+1$ pairs. The momenta obtained by fusing different pairs are connected to a "black box". Its internal structure is not essential for the final result. However, to fix the notations, we will choose it in a particular way and parameterize it by $g-2$ internal momenta $p_{1}^{\prime}, \ldots, p_{g-2}^{\prime}$.

As explained in Section 4, summation of conformal blocks over integer shifts of momenta gives an isomonodromic tau function of the Schlesinger system,

$$
\tau(z)=\sum_{n \in \mathbb{Z}^{g}} \sum_{n^{\prime} \in \mathbb{Z}^{g-1}} \mathcal{B}\left(m\left|p+n, p^{\prime}+n^{\prime}\right| z\right) e^{i n \cdot \tau+i n^{\prime} \cdot \tau^{\prime}} .
$$

The variables $p, p^{\prime}, \tau, \tau^{\prime}$ provide a set of local coordinates on the $(4 g-2)$-dimensional space of monodromy data.

Let us impose a free-field-like conservation constraint on momenta of the unshifted conformal block at each vertex inside the box. These conditions determine the black box momenta $p^{\prime}=$ $p^{\prime}[p]$ in terms of $p$. Explicitly,

$$
p_{k}^{\prime}[p]=p_{k-1}^{\prime}[p]+p_{k+1}, \quad p_{0}^{\prime}[p] \equiv p_{1} .
$$


Also, for $k=1, \ldots, g-1$ we define

$$
\ell_{k}=n_{k}^{\prime}-n_{k-1}^{\prime}-n_{k+1}, \quad n_{0}^{\prime} \equiv n_{1}
$$

Since Barnes $G$-function vanishes at non-positive integer values of the argument, the form of the normalization coefficient (4.42) restricts the sum (6.74) to the domain $\ell_{1}, \ldots, \ell_{g-1} \geq 0$. In the limit

$$
\begin{aligned}
\tau_{j} & \rightarrow-i \infty, \quad \tau_{k}^{\prime} \rightarrow i \infty, \\
\tau_{j}+\sum_{k=j}^{g} \tau_{k-1}^{\prime} & \rightarrow \xi_{j}, \quad \tau_{0}^{\prime} \equiv 0, \quad j=1, \ldots, g,
\end{aligned}
$$

this sum further reduces to the values $\ell_{1}=\ldots=\ell_{g-1}=0$. We thus get a $2 g$-parameter family of tau functions

$$
\tau(z)=\sum_{n \in \mathbb{Z}^{g}} \mathcal{B}\left(m\left|p+n, p^{\prime}[p+n]\right| z\right) e^{i n \cdot \xi} .
$$

Notice that at each of $g-1$ internal vertices of conformal blocks which appear in (6.76), the corresponding momenta satisfy the same conservation conditions as in the unshifted case.

Conformal blocks of this form with $m=m_{\mathrm{AT}} \equiv\left(\frac{1}{4}, \ldots, \frac{1}{4}\right)$ describe correlation functions of the Ashkin-Teller critical model [ $\mathrm{Za}, \mathrm{ZZ}]$. They can be expressed in terms of certain quantities associated to the hyperelliptic curve $\Sigma$ of genus $g$ defined by

$$
\lambda^{2}=\prod_{k=1}^{2 g+2}\left(y-z_{k}\right) .
$$

Let us fix the canonical homology basis of $a$ - and $b$-cycles on $\Sigma$ as shown in Figure 4 . The $g$-dimensional space of holomorphic 1-forms on $\Sigma$ is spanned by

$$
d \omega_{k}=\frac{y^{k-1} d y}{\lambda}, \quad k=1, \ldots, g
$$

The $g \times g$ matrices of $a$ - and $b$-periods

$$
a_{j k}=\oint_{a_{k}} d \omega_{j}, \quad b_{j k}=\oint_{b_{k}} d \omega_{j},
$$

determine the symmetric period matrix $\Omega=a^{-1} b$ of $\Sigma$. The hyperelliptic Riemann theta function with characteristics $[p, q] \in \mathbb{C}^{2 g}$ is defined as the following series:

$$
\theta[p, q](x \mid \Omega)=\sum_{n \in \mathbb{Z}^{g}} e^{\pi i(n+p) \cdot \Omega \cdot(n+p)+2 \pi i(n+p) \cdot(x+q)} .
$$

Even characteristics $\left[p_{S}, q_{S}\right]$ correspond to its non-trivial half-periods and are indexed by partitions $S=\left\{\left\{z_{\alpha_{1}}, \ldots, z_{\alpha_{g+1}}\right\},\left\{z_{\beta_{1}}, \ldots, z_{\beta_{g+1}}\right\}\right\}$ of the set of ramification points into two subsets of equal size. 


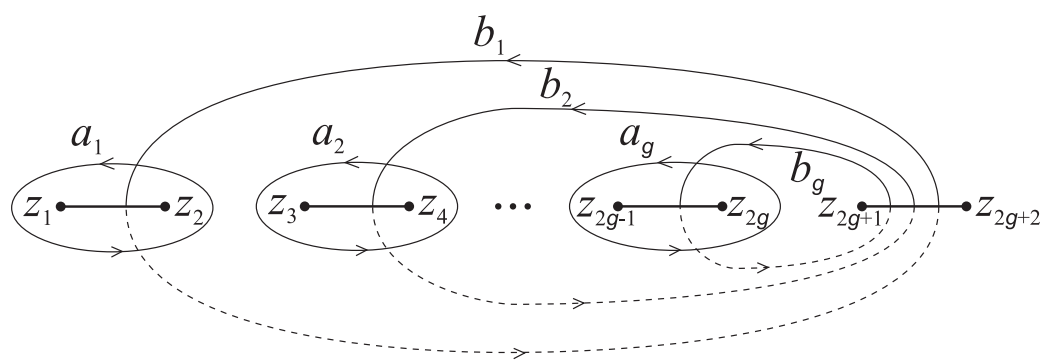

Figure 4: Canonical homology basis on $\Sigma$.

In this notation, the Ashkin-Teller conformal block is given by [Za, ZZ]

$$
\begin{aligned}
& \mathcal{B}\left(m_{\mathrm{AT}}\left|p, p^{\prime}(p)\right| z\right)=\mathcal{G}(p) \mathcal{K}(z) \frac{e^{i \pi p \cdot \Omega \cdot p}}{\theta\left[p_{S}, q_{S}\right](0 \mid \Omega)} \\
& \mathcal{G}(p)=\frac{\cos \pi p_{g-1}^{\prime}}{\pi^{1-g / 2}} \frac{\hat{G}^{2}\left(p_{g-1}^{\prime}+\frac{1}{2}\right)}{\prod_{k=1}^{g} \hat{G}^{2}\left(p_{k}\right)} \\
& \mathcal{K}(z)=\left(\frac{\prod_{j<k}^{g+1}\left(z_{\alpha_{j}}-z_{\alpha_{k}}\right) \prod_{j<k}^{g+1}\left(z_{\beta_{j}}-z_{\beta_{k}}\right)}{\prod_{j, k}^{g+1}\left(z_{\alpha_{j}}-z_{\beta_{k}}\right)}\right)^{\frac{1}{8}} .
\end{aligned}
$$

Here we denote $\hat{G}(p)=\frac{G(1+p)}{G(1-p)}$. The prefactor $\mathcal{G}(p)$ comes from our normalization (4.42) of the chiral vertex operators. Taking into account the recurrence relation $\hat{G}(p+1)=$ $-\pi(\sin \pi p)^{-1} \hat{G}(p)$, we see that the sum (6.76) reduces to the theta function series 6.79), so that

$$
\tau(z)=\mathrm{const} \cdot \mathcal{K}(z) \frac{\theta[p, q](0 \mid \Omega)}{\theta\left[p_{S}, q_{S}\right](0 \mid \Omega)},
$$

with $e^{2 \pi i q_{k}} \equiv-\frac{\sin ^{2} \pi p_{k}}{\cos ^{2} \pi p_{g-1}^{\prime}} e^{i \xi_{k}}$. We thus reproduce the $2 g$-parameter family of tau functions found in $[\overline{\mathrm{KK}}]$. The elliptic case $g=1$ corresponds to Picard solutions of Painlevé VI.

At last let us compute the actual monodromy matrices for $m=m_{\mathrm{AT}}$ applying the rules formulated in Subsection 4.3. Up to overall conjugation, one has

$$
\begin{aligned}
M_{2 k-1} & =\left[C_{[13]}^{k^{\prime}, 1} C^{[k, \ldots, g]} C_{[13]}^{g+1,1}\right]^{-1}\left(B_{1}^{k^{\prime}}\right)^{2} C_{[13]}^{k^{\prime}, 1} C^{[k, \ldots, g]} C_{[13]}^{g+1,1}, \\
M_{2 k} & =\left[C_{[23]}^{k^{\prime}, 0} C^{[k, \ldots, g]} C_{[13]}^{g+1,1}\right]^{-1}\left(B_{2}^{k^{\prime}}\right)^{2} C_{[23]}^{k^{\prime}, 0} C^{[k, \ldots, g]} C_{[13]}^{g+1,1},
\end{aligned}
$$

with $C^{[k, \ldots, g]}=C_{[23]}^{k,-1} C_{[13]}^{k+1,0} \ldots C_{[13]}^{g, 0}$ and $k=2, \ldots, g$. The conservation of momenta at the vertices $k, \ldots, g-1$ implies that all matrices in the product $C^{[k, \ldots, g]}$ are lower triangular. This enables one to explicitly calculate the monodromies in the limit 6.75). Again up to conjugation, the result is

$$
M_{k}=\left(\begin{array}{cc}
0 & i \mu_{k}^{-1} \\
i \mu_{k} & 0
\end{array}\right), \quad k=1, \ldots, 2 g+2
$$


with $\mu_{2 g+1}=e^{2 \pi i p_{g-1}^{\prime}}, \mu_{2 g+2}=1$ and

$$
\mu_{2 k-1}=e^{2 \pi i\left(p_{k-2}^{\prime}+q_{k}\right)}, \quad \mu_{2 k}=-e^{2 \pi i\left(p_{k-1}^{\prime}+q_{k}\right)} .
$$

Note in particular that in the chosen basis the products $M_{2 k-1} M_{2 k}$ and $M_{2 k} M_{2 k+1}$ are given by diagonal matrices, cf [KK, Theorem 3.2].

\section{Outlook}

To conclude we will discuss some further applications and possible directions of future research suggested by our results.

\subsection{Possible applications to the study of $\mathcal{N}=2$ supersymmetric gauge theories}

Our results appear to have interesting implications for the study of a certain class of $4 D \mathcal{N}=2$ supersymmetric gauge theories which is nowadays often called class $\mathcal{S}$. The gauge theories $\mathcal{G}_{C}$ in class $\mathcal{S}$ are associated to Riemann surfaces $C$, possibly with $n$ punctures. The so-called instanton partition functions [LNS, MNS1, MNS2, N, NO] carry important non-perturbative information about the physics of such gauge theories, including the complete description of their low-energy physics via Seiberg-Witten theory [N, NO]. Out of the instanton partition functions one may form the so-called dual instanton partition functions by means of a generalization of the Fourier series [N, $\mathrm{NO}]$.

It was observed in [N, LMN, NO] that the dual instanton partition functions of some supersymmetric gauge theories from class $\mathcal{S}$ have free fermion representations, and therefore represent tau-functions for certain integrable equations. Considerations of the geometric engineering of such gauge theories within string theory have led to the suggestion that the dual instanton partition functions of the gauge theories from class $\mathcal{S}$ should be related to the partition functions of chiral free fermion theories on suitable Riemann surfaces [N, ADKMV, DHSV, DHS] $]^{8}$. More recently it was proposed in [CNO] that the relevant theory of chiral free fermions is defined on the Riemann surface $C$ specifying the gauge theory $\mathcal{G}_{C}$. These relations were called BPS-CFT correspondence in [CNO].

\footnotetext{
${ }^{8}$ The first proposal in this direction was formulated in [N] Section 4.3]. The relations between the topological vertex and free fermion theories discussed in [ADKMV] imply general relations between topological string partition functions of toric Calabi-Yau manifolds, tau-functions and theories of free fermions on certain Riemann surfaces; possible implications for four-dimensional gauge theories were discussed more explicitly in [DHSV], DHS]. In some of the earlier references cited above, it was proposed that the relevant theory of free fermions is defined on the Seiberg-Witten curve $\Sigma$ which for theories of class $\mathcal{S}$ is a branched cover of the curve $C$ defining $\mathcal{G}_{C}$.
} 
In another important recent development it was found that the instanton partition functions of these supersymmetric gauge theories are related to the conformal blocks of the Toda conformal field theories, in the simplest case the Liouville theory [AGT]. The correspondence between instanton partition functions and Liouville conformal blocks is called the AGT-correspondence. However, up to now it was not clear how exactly BPS-CFT-correspondence and AGTcorrespondence are related. Our paper provides a basis for understanding these connections by establishing a direct relation between the conformal field theory of chiral free fermions on a Riemann sphere with $n$ punctures $C_{0, n}$ on the one hand, and the conformal blocks of Liouville theory at $c=1$ on $C_{0, n}$ on the other hand. Our result opens the interesting perspective to derive the $c=1$ case of the AGT-correspondence from the BPS-CFT-correspondence. It would suffice to characterise the relevant $\bar{\partial}_{E}$-operators whose determinants should represent the dual instanton partition function according the BPS-CFT-correspondence more precisely. To this aim it may be convenient to use the language proposed in [DHS]. The connection between the relevant determinants of $\bar{\partial}_{E}$-operators and the isomonodromic tau-functions studied in this paper should then follow from the results of $[\mathrm{P}]$. To complete the derivation of the AGT-correspondence for $c=1$ from the BPS-correspondence it will suffice to observe that the Fourier-transformation appearing in the relation (4.43) between conformal blocks and tau-functions is exactly the transformation from instanton partition functions to the dual instanton partition functions.

\subsection{Verlinde loop operators and quantisation of $\mathcal{M}_{\text {flat }}(C)$}

For $c \neq 1$ one may use the operator-valued monodromies constructed in Section 3.4 to define the so-called Verlinde loop operators [AGGTV, DGOT]. These operators generate a representation of the quantised algebra of algebraic functions on $\mathcal{M}_{\text {flat }}(C)$ on the spaces of Virasoro conformal blocks [TV13]. The definition of the Verlinde loop operators given in [AGGTV, DGOT] can easily be rewritten as deformed traces over products of the operator-valued monodromy matrices defined in Section 3.4 .

In the normalisation for the conformal blocks defined by setting $N\left(\beta_{2}, \alpha, \beta_{1}\right) \equiv 1$ in (3.18) one may analytically continue both the conformal blocks and the corresponding representation of the Verlinde loop operators with respect to the parameter $c$ to generic complex values of this parameter. It is not hard to check that

- the definition of the Verlinde loop operators reduces to taking the ordinary trace of the matrices $\mathrm{M}_{k}$ defined in Section 4.2 at $c=1$,

- the algebra generated by the Verlinde loop operators becomes commutative at this value of the central charge $c$, and 
- the transformation relating Virasoro conformal blocks to tau-functions diagonalizes all Verlinde loop operators simultaneously with eigenvalues being the trace functions (2.5).

We note that the quantum counterparts of the coordinates $(\sigma, \tau)$ that can be defined away from $c=1$ [TV13] remain non-commutative when $c \rightarrow 1$. However, the algebra of all operators that can be constructed from the quantised coordinates $(\sigma, \tau)$ contains the important sub-algebra generated by the Verlinde loop operators. The fact that this sub-algebra becomes commutative for $c=1$ leads to the existence of new representations for the quantised algebra of functions on $\mathcal{M}_{\text {flat }}(C)$ related to the usual one by the transformation defined in Section 4.1. This representation is not unitarily equivalent to the one studied in [TV13] as the measure defining the scalar product for $c>25$, the Liouville three-point function, can not be analytically continued to $c=1$. It should be interesting to investigate this phenomenon and possible generalisations further.

\subsection{Other relations between isomonodromic deformations and Liouville theory}

There are further relations between the isomonodromic deformation problem and Liouville theory: The semiclassical limit of the null-vector decoupling equations in Liouville theory yields Hamilton-Jacobi - like equations that define the Hamiltonians generating the isomonodromic deformation flows. This was first pointed out in [T11], a special case was later rediscovered in [LLNZ].

It seems remarkable that there exist relations between Liouville conformal blocks and isomonodromic tau-functions both in the cases $c=1$ and $c \rightarrow \infty$. A good explanation remains to be found.

Acknowledgements. The present work was supported by the Ukrainian SFFR project F53.2/028, the Program of fundamental research of the physics and astronomy division of NASU, project 01-01-14 of NASU, and the IRSES project "Random and integrable models in mathematical physics".

J.T. would like to thank the Euler Institute (St. Petersburg), where this work was first presented in the workshop "Gauge theories and integrability" for hospitality.

\section{A. Calculation of the trace functions}

Let us compute the trace functions $L_{s}^{r}$ and $L_{t}^{r}$ in terms of the parameters $m_{1 \ldots 4}^{r}, \sigma_{r}, \tau_{r}$ using the algorithm developed in Subsection 3.4.2 along with the rules of Subsection 4.3. The reader is 
referred to Figure $2 \mathrm{~b}$ (with $p$ replaced by $\sigma_{r}$ ) for the labeling of pairs of pants and boundary components.

The trace functions are determined by the classical monodromies around the punctures $z_{1}, z_{2}$, $z_{3}$. To find them explicitly, we first note that the corresponding operator-valued monodromy matrices are given by

$$
\begin{aligned}
& \mathrm{M}_{1}=\left[\mathrm{C}_{[13]}^{R, 0} \mathrm{C}_{[13]}^{L, 1} \cdot \mathrm{C}\right]^{-1}\left(\mathrm{~B}_{1}^{R}\right)^{2} \mathrm{C}_{[13]}^{R, 0} \mathrm{C}_{[13]}^{L, 1} \cdot \mathrm{C}, \\
& \mathrm{M}_{2}=\left[\mathrm{C}_{[23]}^{R,-1} \mathrm{C}_{[13]}^{L, 1} \cdot \mathrm{C}\right]^{-1}\left(\mathrm{~B}_{2}^{R}\right)^{2} \mathrm{C}_{[23]}^{R,-1} \mathrm{C}_{[13]}^{L, 1} \cdot \mathrm{C}, \\
& \mathrm{M}_{3}=\left[\mathrm{C}_{[23]}^{L, 0} \cdot \mathrm{C}\right]^{-1}\left(\mathrm{~B}_{2}^{L}\right)^{2} \mathrm{C}_{[23]}^{L, 0} \cdot \mathrm{C} .
\end{aligned}
$$

Here the common factor $\mathrm{C}$ corresponds to the part of analytic continuation path which relates the base-point $y_{0}$ to the boundary component 3 of $C_{0,3}^{L}$ (the neighborhood of the black dot on the boundary circle in Figure 11). The factor next to it depends on what one wants to achieve at the subsequent step: the black circle on the boundary 2 or the empty circle on the boundary 1 of $C_{0,3}^{L}$. In the latter case, for instance, the arc $[13]_{L}$ should be preceded by the half-turn $b_{3}^{L}$.

The observations of Subsection 4.3 allow one to get rid of the shift operators in the computation of classical monodromies by replacing the operator-valued matrices $\mathrm{C}_{[j i]}^{t, \nu}$ by the ordinary matrices $C_{[j i]}^{t, \nu}$ defined by (4.47). We may therefore set $\mathrm{C}=1$ in the calculation of the trace functions. Also note that the resulting expressions are independent of the parameter $\tau_{4}$ associated to the boundary curve $\delta_{4}$ : this is a consequence of the factorization

$$
(T B)_{i}^{t, \nu}=\left(\tilde{B}_{i}^{t}\right)^{-\nu}\left(\begin{array}{cc}
0 & e^{-\frac{\mathrm{i}}{2} \tau_{i}^{t}} \\
e^{\frac{\mathrm{i}}{2} \tau_{i}^{t}} & 0
\end{array}\right), \quad \tilde{B}_{i}^{t}=\mathrm{i} \sigma_{3} B_{i}^{t} .
$$

We can now write $L_{s}^{r}, L_{t}^{s}$ as the traces

$$
\begin{aligned}
L_{s}^{r} & =\operatorname{tr}\left(\left[C_{[23]}^{R,-1}\right]^{-1}\left(B_{2}^{R}\right)^{2} C_{[23]}^{R,-1}\left[C_{[13]}^{R, 0}\right]^{-1}\left(B_{1}^{R}\right)^{2} C_{[13]}^{R, 0}\right)= \\
& =\operatorname{tr}\left(\left(\tilde{B}_{3}^{R}\right)^{-1} F_{[32]}^{R}\left(\tilde{B}_{2}^{R}\right)^{2} F_{[23]}^{R} \tilde{B}_{3}^{R} F_{[31]}^{R}\left(\tilde{B}_{1}^{R}\right)^{2} F_{[13]}^{R}\right), \\
L_{t}^{r} & =\operatorname{tr}\left(\left[C_{[23]}^{R,-1} C_{[13]}^{L, 1}\right]^{-1}\left(B_{2}^{R}\right)^{2} C_{[23]}^{R,-1} C_{[13]}^{L, 1}\left[C_{[23]}^{L, 0}\right]^{-1}\left(B_{2}^{L}\right)^{2} C_{[23]}^{L, 0}\right) .
\end{aligned}
$$

The first of the equations (2.5) then follows from the easily verified identity

$$
F_{[31]}^{t} \tilde{B}_{1}^{t} F_{[12]}^{t} \tilde{B}_{2}^{t} F_{[23]}^{t} \tilde{B}_{3}^{t}=\mathrm{i},
$$

which should be understood as a version of the Moore-Seiberg hexagonal relation. To demonstrate the second equation, observe that (A.90b may be rewritten as

$$
\begin{gathered}
L_{t}^{r}=G_{+-}^{R} G_{+-}^{L} e^{i \tau_{r}}+\left(G_{++}^{R} G_{--}^{L}+G_{--}^{R} G_{++}^{L}\right)+G_{-+}^{R} G_{-+}^{L} e^{-i \tau_{r}}, \\
G^{R}=\left[F_{[23]}^{R} \tilde{B}_{3}^{R}\right]^{-1}\left(\tilde{B}_{2}^{R}\right)^{2} F_{[23]}^{R} \tilde{B}_{3}^{R}, \quad G^{L}=\tilde{B}_{1}^{L} F_{[12]}^{L}\left(\tilde{B}_{2}^{L}\right)^{2}\left[\tilde{B}_{1}^{L} F_{[12]}^{L}\right]^{-1} .
\end{gathered}
$$

The rest of the computation is straightforward. 


\section{References}

[AGT] L. F. Alday, D. Gaiotto, and Y. Tachikawa, Liouville Correlation Functions from Fourdimensional Gauge Theories, Lett. Math. Phys. 91 (2010) 167-197.

[ADKMV] M. Aganagic, R. Dijkgraaf, A. Klemm, M. Marino, C. Vafa, Topological Strings and Integrable Hierarchies, Commun. Math. Phys. 261 (2006) 451-516

[AGGTV] L. F. Alday, D. Gaiotto, S. Gukov, Y. Tachikawa, H. Verlinde, Loop and surface operators in $\mathcal{N}=2$ gauge theory and Liouville modular geometry, J. High Energy Phys. 1001 (2010) 113.

[CNO] E. Carlsson, N. Nekrasov, A. Okounkov, Five dimensional gauge theories and vertex operators. Preprint arXiv:1308.2465.

[DHSV] R. Dijkgraaf, L. Hollands, P. Sułkowski, C Vafa, Supersymmetric gauge theories, intersecting branes and free fermions JHEP 02 (2008) 106 (doi:10.1088/1126-6708/2008/02/106)

[DHS] R. Dijkgraaf, L. Hollands, P. Sułkowski, Quantum curves and D-modules, JHEP 11 (2009) 047 ( doi:10.1088/1126-6708/2009/11/047)

[DGOT] N. Drukker, J. Gomis, T. Okuda, J. Teschner, Gauge Theory Loop Operators and Liouville Theory, J. High Energy Phys. 1002 (2010) 057.

[GIL] O. Gamayun, N. Iorgov, O. Lisovyy, Conformal field theory of Painlevé VI, J. High Energy Phys. 10 (2012) 038; arXiv:1207.0787[ [hep-th].

[ILT] N. Iorgov, O. Lisovyy, Yu. Tykhyy, Painlevé VI connection problem and monodromy of $c=1$ conformal blocks, J. High Energy Phys. 12 (2013), 029; arXiv:1308.4092 [hep-th].

[Ji] M. Jimbo, Monodromy problem and the boundary condition for some Painlevé equations. Publ. Res. Inst. Math. Sci. 18 (1982), no. 3, 1137-1161.

[KK] A. V. Kitaev, D. A. Korotkin, On solutions of the Schlesinger equations in terms of $\Theta$-functions, Int. Math. Res. Notices 17 (1998) 877-905.

[LLNZ] A. Litvinov, S. Lukyanov, N. Nekrasov, A. Zamolodchikov, Classical Conformal Blocks and Painlevé VI. Preprint arXiv:1309.4700.

[LMN] A. S. Losev, A. Marshakov and N. A. Nekrasov, Small Instantons, Little Strings and Free Fermions. In *Shifman, M. (ed.) et al.: From fields to strings, vol. 1* 581-621 [hep-th/0302191].

[LNS] A.S. Losev, N.A. Nekrasov, S. Shatashvili, Testing Seiberg-Witten solution. Strings, branes and dualities (Cargèse, 1997), 359-372, NATO Adv. Sci. Inst. Ser. C Math. Phys. Sci., 520, Kluwer Acad. Publ., Dordrecht, 1999.

[MNS1] G. Moore, N.A. Nekrasov, S. Shatashvili, Integrating over Higgs branches. Comm. Math. Phys. 209 (2000) 97-121.

[MNS2] G. Moore, N.A. Nekrasov, S. Shatashvili, D-particle bound states and generalized instantons. Comm. Math. Phys. 209 (2000) 77-95.

[MS] G. Moore, N. Seiberg, Classical and quantum conformal field theory, Commun. Math. Phys. 123 (1989) 177-254. 
[N] N. A. Nekrasov, Seiberg-Witten prepotential from instanton counting, Adv. Theor. Math. Phys. 7 (2003) 831-864.

[NO] N. Nekrasov, A. Okounkov, Seiberg-Witten theory and random partitions. The unity of mathematics, 525-596, Progr. Math., 244, Birkhäuser Boston, Boston, MA, 2006.

[NRS] N. Nekrasov, A. Rosly, S. Shatashvili, Darboux coordinates, Yang-Yang functional, and gauge theory, Nucl. Phys. Proc. Suppl. 216 (2011) 69-93; arXiv:1103.3919.

[P] J. Palmer, Determinants of Cauchy-Riemann Operators as $\tau$-Functions. Acta Applicandae Mathematicae 18 (1990) 199-223.

[SMJ79] M. Sato, T. Miwa, M. Jimbo, Holonomic quantum fields. II. The Riemann-Hilbert problem. Publ. Res. Inst. Math. Sci. 15 (1979), no. 1, 201-278.

[SMJ80] M. Sato, T. Miwa, M. Jimbo, Aspects of holonomic quantum fields. Isomonodromic deformation and Ising model. in: "Complex analysis, microlocal calculus and relativistic quantum theory" (Proc. Internat. Colloq., Centre Phys., Les Houches, 1979), pp. 429-491, Lecture Notes in Phys., 126, Springer, Berlin, 1980.

[T03] J. Teschner, A lecture on the Liouville vertex operators, Int. J. Mod. Phys. A19S2 (2004) 436-458

[T11] J. Teschner, Quantization of the Hitchin moduli spaces, Liouville theory and the geometric Langlands correspondence I. Adv. Theor. Math. Phys. 15 (2011), no. 2, 471-564.

[TV13] J. Teschner, G. Vartanov, Supersymmetric gauge theories, quantisation of moduli spaces of flat connections, and conformal field theory. arXiv:1302.3778

[Za] Al. B. Zamolodchikov, Conformal scalar field on the hyperelliptic curve and critical Ashkin-Teller multipoint correlation functions, Nucl. Phys. B285 (1987) 481-503.

[ZZ] A. B. Zamolodchikov, Al. B. Zamolodchikov, Conformal field theory and critical phenomena in two-dimensional systems, MCNMO, Moscow, (2009). 